\title{
Union of Random Minkowski Sums and Network Vulnerability Analysis
}

\author{
Pankaj K. Agarwal • Sariel Har-Peled • \\ Haim Kaplan • Micha Sharir
}

Received: 20 October 2013 / Revised: 30 June 2014 / Accepted: 8 July 2014 /

Published online: 9 September 2014

(C) Springer Science+Business Media New York 2014

\begin{abstract}
Let $\mathcal{C}=\left\{C_{1}, \ldots, C_{n}\right\}$ be a set of $n$ pairwise-disjoint convex sets of constant description complexity, and let $\pi$ be a probability density function (density for short) over the non-negative reals. For each $i$, let $K_{i}$ be the Minkowski sum of $C_{i}$ with a disk of radius $r_{i}$, where each $r_{i}$ is a random non-negative number drawn independently from the distribution determined by $\pi$. We show that the expected complexity of the union of $K_{1}, \ldots, K_{n}$ is $O\left(n^{1+\varepsilon}\right)$ for any $\varepsilon>0$; here the constant of proportionality depends on $\varepsilon$ and the description complexity of the sets in $\mathcal{C}$, but not on $\pi$. If each $C_{i}$ is a convex polygon with at most $s$ vertices, then we show that the expected complexity of the union is $O\left(s^{2} n \log n\right)$. Our bounds hold in a more general model in which we are given an arbitrary multi-set $\Theta=\left\{\theta_{1}, \ldots, \theta_{n}\right\}$ of expansion radii, each a non-negative real number. We assign them to the members of $\mathcal{C}$ by a random permutation, where all permutations are equally likely to be chosen; the expectations are now with respect to these permutations. We also present an application of our results to a problem that arises in analyzing the vulnerability of a network to a physical attack.
\end{abstract}

P. K. Agarwal

Department of Computer Science, Duke University, Box 90129, Durham, NC 27708-0129, USA

e-mail: pankaj@cs.duke.edu

S. Har-Peled

Department of Computer Science, University of Illinois, 201 N. Goodwin Avenue,

Urbana, IL 61801, USA

e-mail: sariel@illinois.edu

H. Kaplan · M. Sharir

School of Computer Science, Tel Aviv University, Tel Aviv 69978, Israel

e-mail: haimk@post.tau.ac.il

M. Sharir

e-mail:michas@post.tau.ac.il 
Keywords Minkowski sum · Arrangement - Network vulnerability · Stochastic model

\section{Introduction}

Union of random Minkowski sums Let $\mathcal{C}=\left\{C_{1}, \ldots, C_{n}\right\}$ be a set of $n$ pairwisedisjoint convex sets of constant description complexity, i.e., the boundary of each $C_{i}$ is defined by a constant number of algebraic arcs of constant maximum degree. Let $D(r)$ denote the disk of radius $r$ centered at the origin. We consider the setup where we are given a sequence $\mathbf{r}=\left\langle r_{1}, \ldots, r_{n}\right\rangle$ of non-negative numbers, called expansion distances (or radii). We set $K_{i}=C_{i} \oplus D\left(r_{i}\right)$, the Minkowski sum of $C_{i}$ with $D\left(r_{i}\right)$. The boundary of $K_{i}$, denoted by $\partial K_{i}$, consists of $O(1)$ algebraic arcs of bounded degree. We refer to the endpoints of the arcs of $\partial K_{i}$ as the vertices of $K_{i}$. If $C_{i}$ is a convex polygon with $s$ vertices, then $\partial K_{i}$ is an alternating concatenation of line segments and circular arcs, where each segment is a parallel shift, by distance $r_{i}$, of an edge of $C_{i}$, and each circular arc is of radius $r_{i}$ and is centered at a vertex of $C_{i}$; see Fig. 1 . Let $\mathcal{K}=\left\{K_{1}, \ldots, K_{n}\right\}$, and let $\mathcal{U}=\mathcal{U}(\mathcal{K})=\bigcup_{i=1}^{n} K_{i}$. The combinatorial complexity of $\mathcal{U}$, denoted by $\psi(\mathcal{C}, \mathbf{r})$, is defined to be the number of vertices of $\mathcal{U}$, each of which is either a vertex of some $K_{i}$ or an intersection point of the boundaries of a pair of $K_{i}$ 's, lying on $\partial \mathcal{~ U . ~ W e ~ d o ~ n o t ~ m a k e ~ a n y ~ a s s u m p t i o n s ~ o n ~ t h e ~ s h a p e ~ a n d ~ l o c a t i o n ~}$ of the sets in $\mathcal{C}$, except for requiring them to be pairwise disjoint.

Our goal is to obtain an upper bound on the expected combinatorial complexity of $\mathcal{U}$, under a suitable probabilistic model for choosing the expansion radii $\mathbf{r}$ of the members of $\mathrm{C}$ - see below for the precise models that we will use.

Network vulnerability analysis Our motivation for studying the above problem comes from the problem of analyzing the vulnerability of a network to a physical attack (e.g., electromagnetic pulse (EMP) attacks, military bombing, or natural disasters [13]), as studied in [2]. Specifically, let $\mathcal{G}=(V, \mathcal{E})$ be a planar graph embedded in the plane, where $V$ is a set of points in the plane and $\mathcal{E}=\left\{e_{1}, \ldots, e_{n}\right\}$ is a set of $n$ segments (often called links) with pairwise-disjoint relative interiors, whose endpoints are points of $V$. For a point $q \in \mathbb{R}^{2}$ and an edge $e$, let $d(q, e)=\min _{p \in e}\|q-p\|$ denote the (minimum) distance between $q$ and $e$. Let $\varphi: \mathbb{R}_{\geq 0} \rightarrow[0,1]$ denote the edge failure probability

Fig. 1 Pairwise-disjoint convex polygons and their Minkowski sums with disks of different radii. The vertices of the union of these sums are highlighted

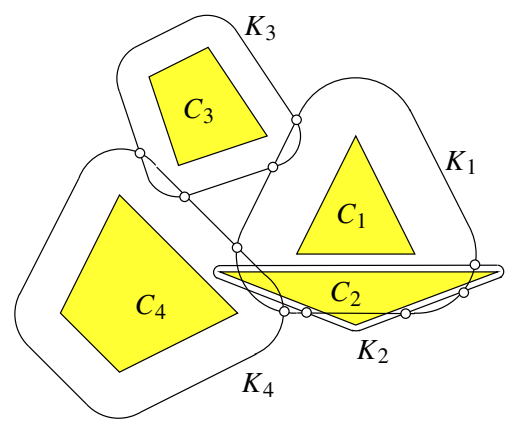




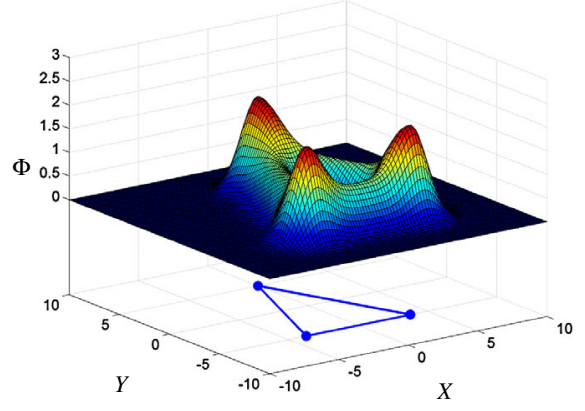

(i)

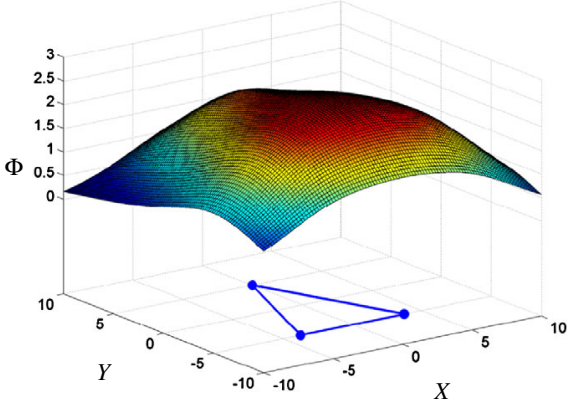

(ii)

Fig. 2 Expected damage for a triangle network and Gaussian probability distribution function with (i) small variance, (ii) large variance

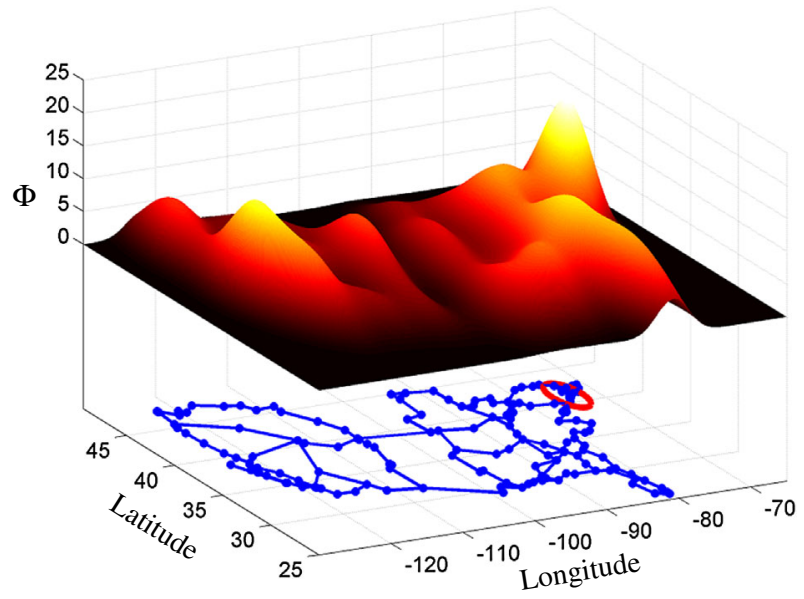

Fig. 3 Expected damage for a complex fiber network. This figure is taken from [2]

function, so that the probability of an edge $e$ to be damaged by a physical attack at a location $q$ is $\varphi(d(q, e))$. In this model, the failure probability only depends on the distance of the point of attack from $e$. We assume that $1-\varphi$ is a cumulative distribution function (cdf), or, equivalently, that $\varphi(0)=1, \varphi(\infty)=0$, and $\varphi$ is monotonically decreasing. A typical example is $\varphi(x)=\max \{1-x, 0\}$, where the cdf is the uniform distribution on $[0,1]$.

For each $e_{i} \in \mathcal{E}$, let $f_{i}(q)=\varphi\left(d\left(q, e_{i}\right)\right)$. The function $\Phi(q, \mathcal{E})=\sum_{i=1}^{n} f_{i}(q)$ gives the expected number of links of $\mathcal{E}$ damaged by a physical attack at a location $q$; see Figs. 2 and 3. Set

$$
\Phi(\mathcal{E})=\max _{q \in \mathbb{R}^{2}} \Phi(q, \mathcal{E})
$$

Our ideal goal is to compute $\Phi(\mathcal{E})$ and a location $q^{*}$ such that $\Phi\left(q^{*}, \mathcal{E}\right)=\Phi(\mathcal{E})$. We refer to such a point $q^{*}$ as a most vulnerable location for $\mathcal{G}$. As evident from Fig. 3 , the 
Fig. 4 A bad choice of expansion distances may cause $\mathcal{U}$ to have quadratic complexity

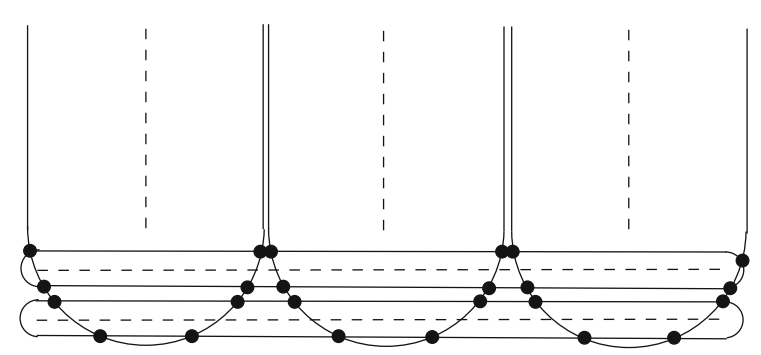

function $\Phi$ can be quite complex, and it is generally hard to compute $\Phi(\mathcal{E})$ exactly, so we focus on computing it approximately. More precisely, given an error parameter $\delta>0$, we seek a point $\tilde{q} \in \mathbb{R}^{2}$ for which $\Phi(\tilde{q}, \mathcal{E}) \geq(1-\delta) \Phi(\mathcal{E})$ (a so-called approximately most vulnerable location). Agarwal et al. [2] proposed a Monte Carlo algorithm for this task. As it turns out, the problem can be reduced to the problem of estimating the maximum depth in an arrangement of random Minkowski sums of the form considered above, under the density model (one of the models for the random choice of expansion distances that we use, which is defined later in the introduction), and its performance then depends on the expected complexity of $\mathcal{U}(\mathcal{K})$. Here $\mathcal{K}$ is a collection of Minkowski sums of the form $e_{i} \oplus D\left(r_{i}\right)$, for a sample of edges $e_{i} \in \mathcal{E}$ and for suitable random choices of the $r_{i}$ 's, from the distribution $1-\varphi$. We adapt and simplify the algorithm in [2] and prove a better bound on its performance by using the sharp (near-linear) bound on the complexity of $\mathcal{U}(\mathcal{K})$ that we derive in this paper; see below and Sect. 5 for details.

Related work (i) Union of geometric objects There is extensive work on bounding the complexity of the union of a set of geometric objects, especially in $\mathbb{R}^{2}$ and $\mathbb{R}^{3}$, and optimal or near-optimal bounds have been obtained for many interesting cases. We refer the reader to the survey paper by Agarwal et al. [5] for a comprehensive summary of most of the known results on this topic. For a set of $n$ planar objects, each of constant description complexity, the complexity of their union can be $\Theta\left(n^{2}\right)$ in the worst case, but many linear or near-linear bounds are known for special restricted cases. For example, a classical result of Kedem et al. [18] asserts that the union of a set of pseudo-disks in $\mathbb{R}^{2}$ has linear complexity. It is also shown in [18] that the Minkowski sums of a set of pairwise-disjoint planar convex objects with a fixed common convex set is a family of pseudo-disks. Hence, in our setting, if all the $r_{i}$ 's were equal, the result of [18] would then imply that the complexity of $\mathcal{U}(\mathcal{K})$ is $O(n)$. On the other hand, an adversarial choice of the $r_{i}$ 's may result in a union $\mathcal{U}$ with $\Theta\left(n^{2}\right)$ complexity; see Fig. 4. Another observation is that if all the distances $r_{i}$ are sufficiently large, the expanded sets will all be fat (see, e.g., [5] for details), in which case $\mathcal{U}(\mathcal{K})$ has near-linear complexity.

(ii) Network vulnerability analysis Most of the early work on network vulnerability analysis considered a small number of isolated, independent failures; see, e.g., [8,23] and the references therein. Since physical networks rely heavily on their physical infrastructure, they are vulnerable to physical attacks such as electromagnetic pulse (EMP) attacks as well as natural disasters [13,27], not to mention military bombing and other similar kinds of attack. This has led to recent work on analyzing the vulnerability 
of a network under geographically correlated failures due to a physical attack at a single location [1,2,21,22,27]. Most papers on this topic have studied a deterministic model for the damage caused by such an attack, which assumes that a physical attack at a location $x$ causes the failure of all links that intersect some simple geometric region (e.g., a vertical segment of unit length, a unit square, or a unit disk) centered at $x$. The impact of an attack is measured in terms of its effect on the connectivity of the network, (e.g., how many links fail, how many pairs of nodes get disconnected, etc.), and the goal is to find the location of attack that causes the maximum damage to the network. In the simpler model studied in [2] and in the present paper, the damage is measured by the number of failed links. This kind of problem is something that both attackers and planners of such networks would like to solve. The former for obvious reasons, and the latter for identifying the most vulnerable portions of the network, in order to protect them better.

In practice, though, it is hard to be certain in advance whether a link will fail by a nearby physical attack. To address this situation, Agarwal et al. [2] introduced the simple probabilistic framework for modeling the vulnerability of a network under a physical attack, as described above. One of the problems that they studied is to compute the largest expected number of links damaged by a physical attack. They described an approximation algorithm for this problem whose expected running time is quadratic in the worst case. A major motivation for the present study is to improve the efficiency of this algorithm and to somewhat simplify it at the same time.

Finally, we note that the study in this paper has potential applications in other contexts, where one wishes to analyze the combinatorial and topological structure of the Minkowski sums (or rather convolutions) of a set of geometric objects (or a function over the ambient space) with some kernel function (most notably a Gaussian kernel), or to perform certain computations on the resulting configuration. Problems of this kind arise in many applications, including statistical learning, computer vision, and robotics; see, e.g., [12,19] and references therein.

Our models We consider two probabilistic models for choosing the sequence $\mathbf{r}=$ $\left\langle r_{1}, \ldots, r_{n}\right\rangle$ of expansion distances:

(i) The density model We are given an arbitrary density (or a probability mass function) $\pi$ over the non-negative reals; for each $1 \leq i \leq n$, we take $r_{i}$ to be a random value drawn independently from the distribution determined by $\pi$.

(ii) The permutation model We are given a multi-set $\Theta=\left\{\theta_{1}, \ldots, \theta_{n}\right\}$ of $n$ arbitrary non-negative real numbers. We draw a random permutation $\sigma$ on $[1: n]$, where all permutations are equally likely to be chosen, and assign $r_{i}:=\theta_{\sigma(i)}$ to $C_{i}$ for each $i=1, \ldots, n$.

Our goal is to prove sharp bounds on the expected complexity of the union $\mathcal{U}(\mathcal{K})$ under these two models. More precisely, for the density model, let $\psi(\mathcal{C}, \pi)$ denote the expected value of $\psi(\mathcal{C}, \mathbf{r})$ (recall that $\psi(\mathcal{C}, \mathbf{r})$ is the complexity of $\mathcal{U}(\mathcal{K})$ under a specific assignment sequence $\mathbf{r}$ of expansion distances), where the expectation is taken over the random choices of $\mathbf{r}$, made from $\pi$ in the manner specified above. Set $\psi(\bigodot)=\max \psi(\mathcal{C}, \pi)$, where the maximum is taken over all probability density (mass) functions. For the permutation model, in an analogous manner, we let $\psi(\mathcal{C}, \Theta)$ denote the expected value of $\psi(\mathcal{C}, \mathbf{r})$, where the expectation is taken over the choices of $\mathbf{r}$, 
obtained by randomly shuffling the members of $\Theta$. Then, with a slight overloading of the notation, we define $\psi(\mathcal{C})=\max \psi(\mathcal{C}, \Theta)$, where the maximum is over all possible choices of the multi-set $\Theta$. We wish to obtain an upper bound on $\psi(\mathcal{C})$ under both models.

We note that the permutation model is more general than the density model, in the sense that an upper bound on $\psi(\mathcal{C})$ under the permutation model immediately implies the same bound on $\psi(\mathcal{C})$ under the density model. Indeed, consider some given density $\pi$, out of whose distribution the distances $r_{i}$ are to be sampled (in the density model). Interpret such a random sample as a 2-stage process, where we first sample from the distribution of $\pi$ a multi-set $\Theta$ of $n$ such distances, in the standard manner of independent repeated draws, and then assign the elements of $\Theta$ to the sets $C_{i}$ using a random permutation (it is easily checked that this reinterpretation does not change the probability space). Let $\mathbf{r}$ be the resulting sequence of expansion radii for the members of $\mathcal{C}$. Using the new interpretation, the expectation of $\psi(\mathcal{C}, \mathbf{r})$ (under the density model), conditioned on the fixed $\Theta$, is at most $\psi(\mathcal{C})$ under the permutation model. Since this bound holds for every $\Theta$, the unconditional expected value of $\psi(\mathcal{C}, \mathbf{r})$ (under the density model) is also at most $\psi(\mathcal{C})$ under the permutation model. Since this holds for every density, the claim follows.

We do not know whether the opposite inequality also holds. A natural reduction from the permutation model to the density model would be to take the input set $\Theta$ of the $n$ expansion distances and regard it as a discrete mass distribution (where each of its members can be picked with probability $1 / n$ ). But then, since the draws made in the density model are independent, most of the draws will not be permutations of $\Theta$, so this approach will not turn $\psi(\mathcal{C})$ under the density model into an upper bound for $\psi(\mathcal{C})$ under the permutation model.

Our results The main results of this paper are near-linear upper bounds on $\psi(\mathcal{C})$ under the two models discussed above. Since the permutation model is more general, in the sense made above, we state, and prove, our results in this model only. We obtain (in Sect. 2) the following bound for the general case.

Theorem 1 Let $\mathcal{C}=\left\{C_{1}, \ldots, C_{n}\right\}$ be a set of $n$ pairwise-disjoint convex sets of constant description complexity in $\mathbb{R}^{2}$. Then the value of $\psi(\mathcal{C})$ under the permutation model, for any multi-set $\Theta$ of expansion radii, is $O\left(n^{1+\varepsilon}\right)$, for any $\varepsilon>0$; the constant of proportionality depends on $\varepsilon$ and the description complexity of the members of $\mathrm{C}$, but not on $\Theta$.

If $\mathcal{C}$ is a collection of convex polygons, we obtain a slightly improved bound by using a different, more geometric argument.

Theorem 2 Let $\mathrm{C}=\left\{C_{1}, \ldots, C_{n}\right\}$ be a set of $n$ pairwise-disjoint convex polygons in $\mathbb{R}^{2}$, where each $C_{i}$ has at most $s$ vertices. Then the maximum value of $\psi(\mathcal{C})$ under the permutation model, for any multi-set $\Theta$ of expansion radii, is $O\left(s^{2} n \log n\right)$; the constant of proportionality that does not depend on $\Theta$.

For simplicity, we first prove Theorem 2 in Sect. 3 for the special case where $\mathcal{C}$ is a set of $n$ segments with pairwise-disjoint relative interiors. Then we extend the proof, in a reasonably straightforward manner, to polygons in Sect. 4. The version involving 
segments admits a somewhat cleaner proof, and is sufficient for the application to network vulnerability analysis.

Using the Clarkson-Shor argument [10], we also obtain the following corollary, which will be needed for the analysis in Sect. 5 .

Corollary 1 Let $\mathcal{C}=\left\{C_{1}, \ldots, C_{n}\right\}$ be a set of $n$ pairwise-disjoint convex sets of constant description complexity. Let $r_{1}, \ldots, r_{n}$ be the random expansion distances, obtained under the permutation model, for any multi-set $\Theta$ of expansion radii, that are assigned to $C_{1}, \ldots, C_{n}$, respectively, and set $\mathcal{K}=\left\{C_{i} \oplus D\left(r_{i}\right) \mid 1 \leq i \leq n\right\}$. Then, for any $1 \leq k \leq n$, the expected number of vertices in the arrangement $\mathcal{A}(\mathcal{K})$ whose depth is at most $k$ is $O\left(n^{1+\varepsilon} k^{1-\varepsilon}\right)$, for any $\varepsilon>0$; the constant of proportionality depends on $\varepsilon$ and the description complexity of the members of $\mathcal{C}$ but not on $\Theta$. If each $C_{i}$ is a convex polygon with at most $s$ vertices, then the bound improves to $O\left(s^{2} n k \log (n / k)\right)$.

We note that the variants of Theorem 1 , Theorem 2 , and Corollary 1 under the density model have identical statements, except that now the constants of proportionality are independent of the density function $\pi$.

Using Theorem 2 and Corollary 1, we present (in Sect. 5) an efficient Monte-Carlo $\delta$-approximation algorithm for computing an approximately most vulnerable location for a network, as defined earlier. Our algorithm is a somewhat simpler, and considerably more efficient, variant of the algorithm proposed by Agarwal et al. [2], and the general approach is similar to the approximation algorithms presented in $[3,4,6]$ for computing the depth in an arrangement of a set of objects. Specifically, we establish the following result.

Theorem 3 Given a set $\mathcal{E}$ of $n$ segments in $\mathbb{R}^{2}$ with pairwise-disjoint relative interiors, an edge-failure-probability function $\varphi$ such that $1-\varphi$ is a cdf, and a constant $0<\delta<1$, one can compute, in $O\left(\delta^{-4} n \log ^{3} n\right)$ time, a location $\tilde{q} \in \mathbb{R}^{2}$, such that $\Phi(\tilde{q}, \mathcal{E}) \geq(1-\delta) \Phi(\mathcal{E})$ with probability at least $1-1 / n^{c}$, for arbitrarily large $c$; the constant of proportionality in the running-time bound depends on $c$.

\section{The Case of Convex Sets}

In this section we prove Theorem 1 . We have a collection $\mathcal{C}=\left\{C_{1}, \ldots, C_{n}\right\}$ of $n$ pairwise-disjoint compact convex sets in the plane, each of constant description complexity. Let $\Theta$ be a multi-set of $n$ non-negative real numbers $0 \leq \theta_{1} \leq \theta_{2} \leq$ $\cdots \leq \theta_{n}$. We choose a random permutation $\sigma$ of $[1: n]$, where all permutations are equally likely to be chosen, put $r_{i}=\theta_{\sigma(i)}$ for $i=1, \ldots, n$, and form the Minkowski sums $K_{i}=C_{i} \oplus D\left(r_{i}\right)$, for $i=1, \ldots, n$. We put $\mathcal{K}=\left\{K_{1}, \ldots, K_{n}\right\}$. We prove a near-linear upper bound on the expected complexity of $\mathcal{U}(\mathcal{K})$, as follows.

Fix a parameter $t$, whose value will be determined later, and put $\rho=\theta_{n-t}$, the $(t+1)$-st largest distance in $\Theta$. Put $\mathrm{C}^{+}=\left\{C_{i} \in \mathcal{C} \mid \sigma(i)>n-t\right\}$ and $\mathrm{C}^{-}=\left\{C_{i} \in \mathcal{C} \mid \sigma(i) \leq n-t\right\}$. That is, $\mathrm{C}^{+}$is the set of the $t$ members of $\mathrm{C}$ that were assigned the $t$ largest distances in $\Theta$, and $\mathrm{C}^{-}$is the complementary subset.

By construction, $\mathrm{C}^{+}$is a random subset of $\mathcal{C}$ of size $t$ (where all $t$-element subsets of $\mathcal{C}$ are equally likely to arise as $\mathrm{C}^{+}$). Moreover, conditioned on the choice of $\mathrm{C}^{+}$, the set $\mathcal{C}^{-}$is fixed, and the subset $\Theta^{-}$of the $n-t$ distances in $\Theta$ that are assigned to 
them is also fixed. Furthermore, the permutation that assigns the elements of $\Theta^{-}$to the sets in $\mathrm{C}^{-}$is a random permutation.

For each $C_{i} \in \mathcal{C}^{+}$, put $K_{i}^{*}=C_{i} \oplus D(\rho)$. Put $\left(\mathcal{K}^{*}\right)^{+}=\left\{K_{i}^{*} \mid C_{i} \in \mathcal{C}^{+}\right\}$, and let $\mathcal{U}^{*}$ denote the union of $\left(\mathcal{K}^{*}\right)^{+}$. Note that $\mathcal{U}^{*} \subseteq \mathcal{U}$, because $K_{i}^{*} \subseteq K_{i}$ for each $C_{i} \in \mathrm{e}^{+}$. Since the $C_{i}$ 's are pairwise-disjoint and we now add the same disk to each of them, $\left(\mathcal{K}^{*}\right)^{+}$is a collection of pseudo-disks [18], and therefore $\mathcal{U}^{*}$ has $O(t)$ complexity. More precisely, $\partial \mathcal{U}^{*}$ has at most $6 t-12$ vertices that are intersection points of boundaries of pairs of sets of $\left(\mathcal{K}^{*}\right)^{+}[18]$.

Let $\mathcal{V}$ denote the vertical decomposition of the complement of $\mathcal{U}^{*}$; it consists of $O(t)$ pseudo-trapezoids, each defined by at most four elements of $\left(\mathcal{K}^{*}\right)^{+}$(that is, of $\mathrm{C}^{+}$). See [25] for more details concerning vertical decompositions. For short, we refer to these pseudo-trapezoids as trapezoids.

In a similar manner, for each $C_{i} \in \mathrm{C}^{-}$, define $K_{i}^{*}=C_{i} \oplus D(\rho)$; note that $K_{i} \subseteq K_{i}^{*}$ for each such $i$. Put $\left(\mathcal{K}^{*}\right)^{-}=\left\{K_{i}^{*} \mid C_{i} \in \mathcal{C}^{-}\right\}$. Since $\mathcal{C}^{+}$is a random sample of $\mathcal{C}$ of size $t$, the following lemma follows from a standard random-sampling argument; see [20, Section 4.6] for a proof.

Lemma 1 With probability at least $1-O\left(\frac{1}{n^{c-4}}\right)$, every (open) trapezoid $\tau$ of $\mathcal{V}$ intersects at most $k:=\frac{c n}{t} \ln n$ of the sets of $\left(\mathcal{K}^{*}\right)^{-}$, for sufficiently large $c>4$.

For each trapezoid $\tau$ of $\mathcal{V}$, let $\mathrm{C}_{\tau}^{-}$denote the collection of the sets $C_{i} \in \mathrm{C}^{-}$for which $K_{i}^{*}$ intersects $\tau$. We form the union $\mathcal{U}_{\tau}^{-}$of the "real" (and smaller) corresponding sets $K_{i}$, for $C_{i} \in \mathcal{C}_{\tau}^{-}$, and clip it to within $\tau$, i.e., keep only its portion with $\tau$ (clearly, no other set $C_{i} \in \mathrm{C}^{-}$can have its real expansion $K_{i}$ meet $\tau$ ). Finally, we take all the "larger" sets $C_{i} \in \mathrm{C}^{+}$, and form the union of $\mathcal{U}_{\tau}^{-}$with the corresponding "real" $K_{i}$ 's, again clipping it to within $\tau$. The overall union $\mathcal{U}$ is the union of $\mathcal{U}^{*}$ and of all the modified unions $\mathcal{U}_{\tau}^{-}$, for $\tau \in \mathcal{V}$.

This divide-and-conquer process leads to the following recursive estimation of the expected complexity of $\mathcal{U}$. For $m \leq n$, let $\mathcal{C}^{\prime}$ be any subset of $m$ sets of $\mathcal{C}$, and let $\Theta^{\prime}$ be any subset of $m$ elements of $\Theta$, which we enumerate, with some abuse of notation, as $\theta_{1}, \ldots, \theta_{m}$. Let $T\left(\mathfrak{C}^{\prime}, \Theta^{\prime}\right)$ denote the expected complexity of the union of the expanded regions $C_{i} \oplus D\left(\theta_{\sigma^{\prime}(i)}\right)$, for $C_{i} \in \mathrm{C}^{\prime}$, where the expectation is over the random shuffling permutation $\sigma^{\prime}$ (on $[1: m]$ ). Let $T(m)$ denote the maximum value of $T\left(\complement^{\prime}, \Theta^{\prime}\right)$, over all subsets $\varrho^{\prime}$ and $\Theta^{\prime}$ of size $m$ each, as just defined.

Let us first condition the analysis on a fixed choice of $\mathrm{C}^{+}$. That is, we condition the analysis on the subset of those permutations $\sigma$ that assign the $t$ largest distances in $\Theta$ to the sets of $\mathcal{C}^{+}$. This determines $\mathcal{U}^{*}$ and $\mathcal{V}$ uniquely. Hence we have a fixed set of trapezoids, and for each trapezoid $\tau$ we have a fixed set $\mathcal{C}_{\tau}^{-}$of $k_{\tau}=\left|\mathcal{C}_{\tau}^{-}\right|$sets, whose expansions by $\rho$ meet $\tau$. Moreover, choosing $c=6$ in Lemma 1 , with probability $1-O\left(\frac{1}{n^{2}}\right)$ our $\mathrm{C}^{+}$is such that $k_{\tau} \leq \frac{c n}{t} \ln n$ for each $\tau \in \mathcal{V}$. Assume for now that $\mathrm{C}^{+}$does indeed have this property. The set $\Theta_{\tau}^{-}$of distances assigned to these sets is not fixed, but it is a random subset of $\left\{\theta_{1}, \ldots, \theta_{n-t}\right\}$ of size $k_{\tau} \leq \frac{c n}{t} \ln n$, where $k_{\tau}$ depends only on $\tau$. Moreover, the assignment (under the original random permutation $\sigma$ ) of these distances to the sets in $\mathcal{C}_{\tau}^{-}$is a random permutation. Hence, conditioning further on the choice of $\Theta_{\tau}^{-}$, the expected complexity of $\mathcal{U}_{\tau}^{-}$, before its modification by the expansions of the larger sets of $\mathcal{C}^{+}$, and ignoring its clipping to within $\tau$, is 


$$
T\left(\mathcal{C}_{\tau}^{-}, \Theta_{\tau}^{-}\right) \leq T\left(k_{\tau}\right) \leq T\left(\frac{c n}{t} \ln n\right)
$$

Hence the last expression also bounds the unconditional expected complexity of the unmodified and unclipped $\mathcal{U}_{\tau}^{-}$(albeit still conditioned on the choice of $\mathcal{C}^{+}$). Summing this over all $O(t)$ trapezoids $\tau$ of $\mathcal{V}$, we get a bound of at most

$$
\operatorname{at} T\left(\frac{c n}{t} \ln n\right)
$$

for a suitable constant $a$. To this we need to add the number of intersections between the boundaries of the unions $\mathcal{U}_{\tau}^{-}$with the respective trapezoid boundaries, and the number of intersections between the boundaries of the $t$ larger expansions and the boundaries of all the expansions, that appear on the boundary of $\mathcal{U}$. The last two quantities are clearly $O(n t)$ (the constant in this latter expression depends on the description complexity of the sets in $\mathcal{C}$ ). Altogether, we obtain that, with probability $1-O\left(\frac{1}{n^{2}}\right)$, the complexity of $\mathcal{U}(\mathcal{K})$ is at most (reusing the constant $a$ for simplicity)

$$
a t T\left(\frac{c n}{t} \ln n\right)+a n t
$$

which holds when $n$ is sufficiently large. With probability $O\left(\frac{1}{n^{2}}\right), \mathrm{e}^{+}$may fail to satisfy the uniform bound $k_{\tau} \leq \frac{6 n}{t} \ln n$, but, since the complexity of $\mathcal{U}(\mathcal{K})$ is always $O\left(n^{2}\right)$, the contribution of these "bad" choices of $\mathrm{C}^{+}$to the overall expectation is $O(1)$. In other words, when $n$ is at least some sufficiently large constant $n_{0}$, we get the recurrence

$$
T(n) \leq a t T\left(\frac{c n}{t} \ln n\right)+a n t+a,
$$

and when $n$ is smaller than $n_{0}$, we use the trivial bound $T(n)=O\left(n^{2}\right)$.

The solution of this recurrence is $T(n) \leq A n^{1+\varepsilon}$, for any $\varepsilon>0$, with an appropriate choice of parameters, where $A$ depends on $\varepsilon$ and on the other constants appearing in the recurrence. Indeed, fix the value of $\varepsilon$, and set $t=n^{\varepsilon / 2}$. Substituting $T\left(n^{\prime}\right) \leq A\left(n^{\prime}\right)^{1+\varepsilon}$ in the right-hand side of (1), for $n^{\prime}=\frac{c n}{t} \ln n<n$, an assumption that holds for $n \geq n_{0}$ and $n_{0}$ sufficiently large, we get that

$$
\begin{aligned}
T(n) & \leq a n^{\varepsilon / 2} A\left(c n^{1-\varepsilon / 2} \ln n\right)^{1+\varepsilon}+a n^{1+\varepsilon / 2}+a \\
& =A\left(a c^{1+\varepsilon}\right) \cdot n^{1+\varepsilon-\varepsilon^{2} / 2}(\ln n)^{1+\varepsilon}+a n^{1+\varepsilon / 2}+a,
\end{aligned}
$$

which is smaller than $A n^{1+\varepsilon}$ for sufficiently large choices of $A$ and $n_{0}$ (which depend on $\varepsilon$ ). Specifically, $n_{0}$ should be such that $n^{\varepsilon^{2} / 2}>2 a c^{1+\varepsilon}(\ln n)^{1+\varepsilon}$ for all $n \geq n_{0}$. For this choice, a sufficiently large choice of $A$ ensures that the right-hand side is indeed at most $A n^{1+\varepsilon}$. Finally, by choosing $A$ sufficiently large we can also ensure that the bound $O\left(n^{2}\right)$, for any $n \leq n_{0}$, is at most $A n^{1+\varepsilon}$. 
Remark It might be possible to improve the bound on the expected complexity of $\mathcal{U}(\mathcal{K})$ to $O(n$ polylog $(n))$ by replacing the above divide-and-conquer approach with a randomized incremental construction, as in [15].

\section{The Case of Segments}

Let $\mathcal{E}=\left\{e_{1}, \ldots, e_{n}\right\}$ be a collection of $n$ line segments in the plane with pairwisedisjoint relative interiors, and as in Sect. 2, let $\Theta$ be a multi-set of $n$ non-negative real numbers $0 \leq \theta_{1} \leq \theta_{2} \leq \cdots \leq \theta_{n}$. For simplicity, we assume that the segments in $\mathcal{E}$ are in general position, i.e., no segment is vertical, no two of them share an endpoint, and no two are parallel. Moreover, we assume that the expansion distances in $\Theta$ are positive and in "general position" with respect to $\mathcal{E}$, so as to ensure that, no matter which permutation we draw, the expanded sets of $\mathcal{K}$ are also in general position-no pair of them are tangent and no three have a common boundary point. ${ }^{1}$ Using the standard symbolic perturbation techniques (see, e.g., [25, Chapter 7]), the proof can be extended when $\mathcal{E}$ or $\Theta$ is not in general position or when some of the expansion distances are 0 ; we omit here the routine details.

For each $1 \leq i \leq n$, let $a_{i}, b_{i}$ be the left and right endpoints, respectively, of $e_{i}$ (as mentioned, we assume, with no loss of generality, that no segment in $\mathcal{E}$ is vertical). We draw a random permutation $\sigma$ of $\{1, \ldots, n\}$, and, for each $1 \leq i \leq n$, we put $r_{i}=\theta_{\sigma(i)}$. We then form the Minkowski sums $K_{i}=e_{i} \oplus D\left(r_{i}\right)$, for $i=1, \ldots, n$. We refer to such a $K_{i}$ as a racetrack. Its boundary consists of two semicircles $\gamma_{i}^{-}$and $\gamma_{i}^{+}$, centered at the respective endpoints $a_{i}$ and $b_{i}$ of $e_{i}$, and of two parallel copies, $e_{i}^{-}$and $e_{i}^{+}$, of $e_{i}$; we use $e_{i}^{-}$(resp., $e_{i}^{+}$) to denote the straight edge of $K_{i}$ lying below (resp., above) $e_{i}$. Let $a_{i}^{-}, a_{i}^{+}$(resp., $b_{i}^{-}, b_{i}^{+}$) denote the left (resp., right) endpoints of $e_{i}^{-}, e_{i}^{+}$, respectively. We regard $K_{i}$ as the union of two disks $D_{i}^{-}, D_{i}^{+}$of radius $r_{i}$ centered at the respective endpoints $a_{i}, b_{i}$ of $e_{i}$, and a rectangle $R_{i}$ of width $2 r_{i}$ having $e_{i}$ as a midline. The left endpoint $a_{i}$ splits the edge $a_{i}^{-} a_{i}^{+}$of $R_{i}$ into two segments of equal length, and similarly $b_{i}$ splits the edge $b_{i}^{-} b_{i}^{+}$of $R_{i}$ into two segments of equal lengths. We refer to these four segments $a_{i} a_{i}^{-}, a_{i} a_{i}^{+}, b_{i} b_{i}^{-}, b_{i} b_{i}^{+}$as the portals of $R_{i}$; see Fig. 5(i). Set $\mathcal{K}=\left\{K_{i} \mid 1 \leq i \leq n\right\}, \mathcal{D}=\left\{D_{i}^{+}, D_{i}^{-} \mid 1 \leq i \leq n\right\}$, and $\mathcal{R}=\left\{R_{i} \mid 1 \leq i \leq n\right\}$.

As above, let $\mathcal{U}=\mathcal{U}(\mathcal{K})$ denote the union of $\mathcal{K}$. We show that the expected number of vertices on $\partial \mathcal{U}$ is $O(n \log n)$, where the expectation is over the choice of the random permutation $\sigma$ that produces the distances $r_{1}, \ldots, r_{n}$.

We classify the vertices of $\partial \mathcal{U}$ into three types (see Fig. 5(ii)):

(i) CC-vertices, which lie on two semicircular arcs of the respective pair of racetrack boundaries;

(ii) $R R$-vertices, which lie on two straight-line edges; and

(iii) $C R$-vertices, which lie on a semicircular arc and on a straight-line edge.

\footnotetext{
1 When we carry over the analysis to the density model, the latter assumption will hold with probability 1 when $\pi$ is Lebesgue-continuous, but may fail for a discrete probability mass distribution. In the latter situation, we can use symbolic perturbations to turn $\pi$ into a density in general position, without affecting the asymptotic bound that we are after.
} 


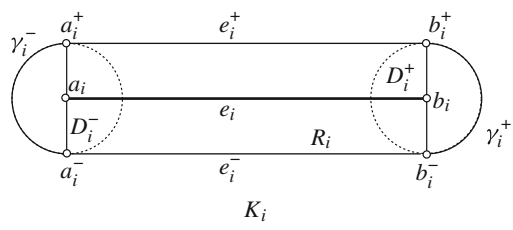

(i)

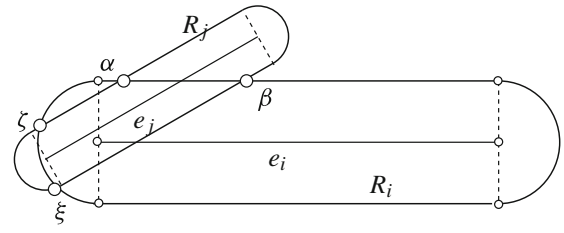

(ii)

Fig. 5 (i) Segment $e_{i}$, racetrack $K_{i}$, and its constituents rectangle $R_{i}$ and disks $D_{i}^{-}, D_{i}^{+}$. (ii) Union of two racetracks. $\alpha, \beta$ are RR-vertices, $\zeta$ is a CR-vertex, and $\xi$ is a CC-vertex; $\alpha$ is a non-terminal vertex and $\beta$ is a terminal vertex (because of the edge of $R_{j}$ it lies on, which ends inside $K_{i}$ )

Bounding the number of CC-vertices is trivial because they are also vertices of $\mathcal{U}(\mathcal{D})$, the union of the $2 n$ disks $D_{i}^{-}, D_{i}^{+}$, so their number is $O(n)[5,18]$. We therefore focus on bounding the expected number of RR- and CR-vertices of $\partial \mathcal{U}$.

\subsection{RR-Vertices}

Let $v$ be an RR-vertex of $\mathcal{U}$, lying on $\partial R_{i}$ and $\partial R_{j}$, the rectangles of two respective segments $e_{i}$ and $e_{j}$. Denote the edges of $R_{i}$ and $R_{j}$ containing $v$ as $e_{i}^{ \pm} \in\left\{e_{i}^{-}, e_{i}^{+}\right\}$ and $e_{j}^{ \pm} \in\left\{e_{j}^{-}, e_{j}^{+}\right\}$, respectively. A vertex $v$ is terminal if either a subsegment of $e_{i}^{ \pm}$ connecting $v$ to one of the endpoints of $e_{i}^{ \pm}$is fully contained in $K_{j}$, or a subsegment of $e_{j}^{ \pm}$connecting $v$ to one of the endpoints of $e_{j}^{ \pm}$is fully contained in $K_{i}$; otherwise $v$ is a non-terminal vertex. For example, in Fig. 5(ii), $\beta$ is a terminal vertex, and $\alpha$ is a non-terminal vertex. There are at most $4 n$ terminal vertices on $\partial \mathcal{U}$, so it suffices to bound the expected number of non-terminal vertices.

Our strategy is first to describe a scheme that charges each non-terminal RR-vertex $v$ to one of the portals of one of the two rectangles of $\mathcal{R}$ on whose boundary $v$ lies, and then to prove that the expected number of vertices charged to each portal is $O(\log n)$. The bound $O(n \log n)$ on the expected number of (non-terminal) RR-vertices then follows.

Let $v$ be a non-terminal RR-vertex lying on $\partial R_{i} \cap \partial R_{j}$, for two respective input segments $e_{i}$ and $e_{j}$. To simplify the notation, we rename $e_{i}$ and $e_{j}$ as $e_{1}$ and $e_{2}$. Note that $v$ is an intersection of $e_{1}^{+}$or $e_{1}^{-}$with $e_{2}^{+}$or $e_{2}^{-}$. Since the analysis is the same for each of these four choices we will say, using the notations already introduced, that $v$ is the intersection of $e_{1}^{ \pm}$with $e_{2}^{ \pm}$where $e_{1}^{ \pm}$(resp., $e_{2}^{ \pm}$) is either $e_{1}^{+}$(resp., $e_{2}^{+}$) or $e_{1}^{-}$ (resp., $e_{2}^{-}$).

For $i=1,2$, let $g_{i}^{ \pm}$denote the (unique) portion of $e_{i}^{ \pm}$between $v$ and an endpoint $w_{i}$ so that, locally near $v, g_{i}^{ \pm}$is contained in the second racetrack $K_{3-i}$ (that is, in $\left.R_{3-i}\right)$. Now take $Q_{i}$ to be the rectangle with $g_{i}^{ \pm}=v w_{i}$ as one of its sides, and with the orthogonal projection of $g_{i}^{ \pm}$onto $e_{i}$ as the opposite parallel side. We denote this edge of $Q_{i}$, which is part of $e_{i}$, by $E_{i}$. We let $A_{i}$ be the side perpendicular to $E_{i}$ and incident to $w_{i}$. Note that $A_{i}$ is one of the four portals of $R_{i}$. We denote by $A_{i}^{*}$ the side of $Q_{i}$ that is parallel to $A_{i}$ (and incident to $v$ ). See Fig. 6. 


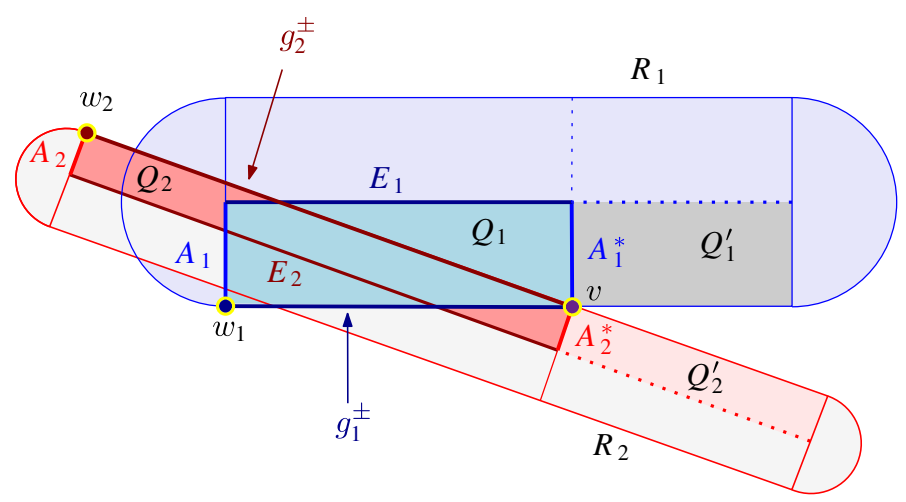

Fig. 6 The rectangles $Q_{1}$ and $Q_{2}$ defined for a non-terminal RR-vertex $v$

Each rectangle $Q_{i}, i=1,2$, has a complementary rectangle $Q_{i}^{\prime}$ on the same side of $e_{i}$, which is openly disjoint from $Q_{i}$, so that $Q_{i} \cup Q_{i}^{\prime}$ is the half of the rectangular portion $R_{i}$, between $e_{i}$ and $e_{i}^{ \pm}$, of the full racetrack $K_{i}$. Again, see Fig. 6 .

Since $E_{i} \subset e_{i}$ for $i=1,2$, it follows that $E_{1}$ and $E_{2}$ are disjoint. Since $v$ is a nonterminal RR-vertex, $w_{1}$ lies outside $K_{2}$ and $w_{2}$ lies outside $K_{1}$. So, as we walk along the edge $v w_{i}$ of $Q_{i}$ from $v$ to $w_{i}$, we enter, locally near $v$, into the other racetrack $K_{3-i}$, but then we have to exit it before we get to $w_{i}$. Note that either of these walks, say from $v$ to $w_{1}$, may enter $Q_{2}$ or keep away from $Q_{2}$ (and enter instead the complementary rectangle $Q_{2}^{\prime}$ ); see Figs. 6 and 8(i) for the former situation, and Fig. 8(ii) for the latter one.

Another important property of the rectangles $Q_{1}$ and $Q_{2}$, which follows from their definition, is that they are "oppositely oriented", when viewed from $v$, in the following sense. When we view from $v$ one of $Q_{1}$ and $Q_{2}$, say $Q_{1}$, and turn counterclockwise, we first see $E_{1}$ and then $A_{1}$, and when we view $Q_{2}$ and turn counterclockwise, we first see $A_{2}$ and then $E_{2}$.

So far our choice of which among the segments defining $v$ is denoted by $e_{1}$ and which is denoted by $e_{2}$ was arbitrary. But in the rest of our analysis we will use $e_{1}$ to denote the segment such that when we view $Q_{1}$ from $v$ and turn counterclockwise, we first see $E_{1}$ and then $A_{1}$. The other segment is denoted by $e_{2}$. (This is indeed the situation depicted in Fig. 6.)

The following lemma provides the key ingredient for our charging scheme.

Lemma 2 Let $v$ be a non-terminal RR-vertex. Then, in the terminology defined above, one of the edges, say $E_{1}$, has to intersect either the portal $A_{2}$ of the other rectangle $Q_{2}$, or the portal $A_{2}^{\prime}$ of the complementary rectangle $Q_{2}^{\prime}$.

Proof For $i=1,2$, we associate with $Q_{i}$ a viewing arc $\Gamma_{i}$, consisting of all orientations of the rays that emanate from $v$ and intersect $Q_{i}$. Each $\Gamma_{i}$ is a quarter-circular arc (of angular span $90^{\circ}$ ), which is partitioned into two subarcs $\Gamma_{i}^{A}, \Gamma_{i}^{E}$, at the orientation at which $v$ sees the opposite vertex of the corresponding rectangle $Q_{i} ; \Gamma_{i}^{A}$ (resp., $\Gamma_{i}^{E}$ ) is the subarc in which we view $A_{i}$ (resp., $E_{i}$ ). See Fig. 7. 
Fig. 7 The two viewing arcs

$\Gamma_{1}, \Gamma_{2}$, their partitions into $\Gamma_{1}^{E}$, $\Gamma_{1}^{A}$ and $\Gamma_{2}^{A}, \Gamma_{2}^{E}$, mnemonically denoted by the respective symbols $A, E$, and their overlap

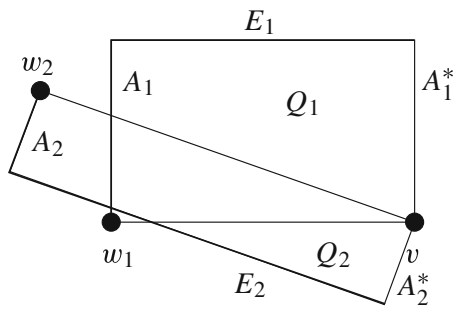

(i)
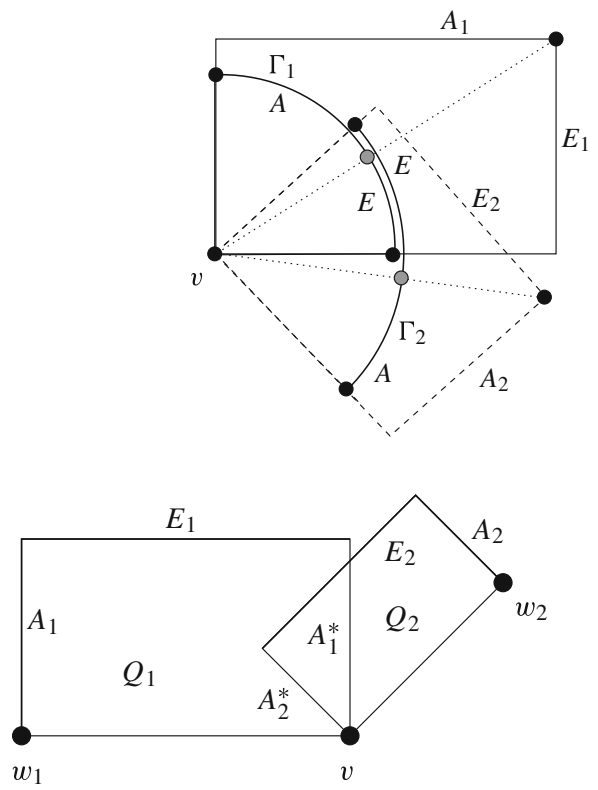

(ii)

Fig. 8 (i) One possible interaction between $Q_{1}$ and $Q_{2}$. The overlap is of type $A A$, the intersection $\Gamma_{0}$ of the viewing arcs is delimited by the orientations of $\overrightarrow{v w_{1}}$ and $\overrightarrow{v w_{2}}$. (ii) Another possible interaction between $Q_{1}$ and $Q_{2}$. The overlap is of type $E E$, the intersection $\Gamma_{0}$ of the viewing arcs does not contain the orientations of $\overrightarrow{v w_{1}}$ and $\overrightarrow{v w_{2}}$

Moreover, the opposite orientations of $Q_{1}$ and $Q_{2}$ mean that, as we trace these arcs in counterclockwise direction, $\Gamma_{1}^{E}$ precedes $\Gamma_{1}^{A}$, whereas $\Gamma_{2}^{E}$ succeeds $\Gamma_{2}^{A}$. That is, the clockwise endpoint of $\Gamma_{1}$ is adjacent to $\Gamma_{1}^{E}$ and we call it the E-endpoint of $\Gamma_{1}$, and the counterclockwise endpoint of $\Gamma_{1}$, called the $A$-endpoint, is adjacent to $\Gamma_{1}^{A}$. Symmetrically, the clockwise endpoint of $\Gamma_{2}$ is its A-endpoint and is adjacent to $\Gamma_{2}^{A}$, and its counterclockwise endpoint is the E-endpoint, adjacent to $\Gamma_{2}^{E}$. See Figs. 7 and 8.

Finally, the overlapping of $Q_{1}$ and $Q_{2}$ near $v$ mean that the arcs $\Gamma_{1}$ and $\Gamma_{2}$ overlap too. Let $\Gamma_{0}:=\Gamma_{1} \cap \Gamma_{2}$.

The viewing arcs $\Gamma_{1}$ and $\Gamma_{2}$ can overlap in one of the following two ways. (Since each arc is of angular span $90^{\circ}$, no other kind of overlap, such as nesting of one arc inside the other, is possible.)

AA-overlap: The clockwise and the counterclockwise endpoints of $\Gamma_{0}$ are the A-endpoints of $\Gamma_{2}$ and $\Gamma_{1}$, respectively. See Fig. 8(i).

EE-overlap: The clockwise and the counterclockwise endpoints of $\Gamma_{0}$ are the E-endpoints of $\Gamma_{1}$ and $\Gamma_{2}$, respectively. See Fig. 8(ii).

We now assume that none of the four intersections (between one of the segments and a suitable portal of the other rectangle), mentioned in the statement of the lemma, occur. We reach a contradiction by showing that under this assumption neither type of overlap can happen. 
Fig. 9 Illustrating the argument that $\rho_{1}$ and $\rho_{2}$ cannot intersect in an AA-overlap

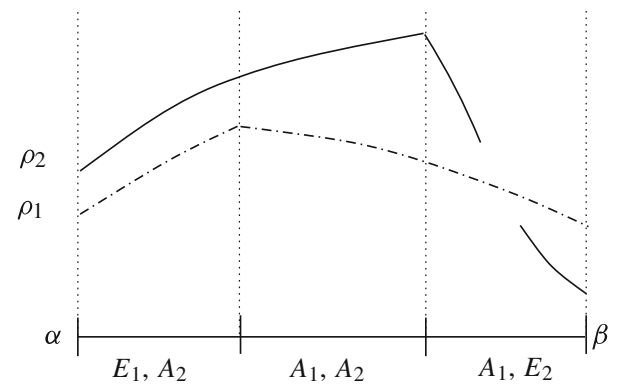

AA-overlap For $i=1,2$, let $\rho_{i}(\theta)$, for $\theta \in \Gamma_{i}$, denote the length of the intersection of $Q_{i}$ with the ray emanating from $v$ in direction $\theta$. Note that $\rho_{i}$ is bimodal: it increases to its maximum, which occurs at the direction to the vertex of $Q_{i}$ opposite to $v$, and then decreases back (each of the two pieces is a simple trigonometric function of $\theta$ ).

Write $\Gamma_{0}=[\alpha, \beta]$. Since the overlap of $\Gamma_{1}$ and $\Gamma_{2}$ is an AA-overlap, $\alpha$ is the orientation of $\overrightarrow{v w_{2}}$, and we have $\rho_{2}(\alpha)>\rho_{1}(\alpha)$ (we have to exit $Q_{1}$ before we reach $\left.w_{2}\right)$. Symmetrically, we have $\rho_{2}(\beta)<\rho_{1}(\beta)$. See Fig. 8(i). Hence, by continuity, there must exist $\theta \in \Gamma_{0}$ where $\rho_{1}(\theta)=\rho_{2}(\theta)$.

We claim however that this equality is impossible. Indeed, by taking into account the partitions $\Gamma_{1}=\Gamma_{1}^{E} \cup \Gamma_{1}^{A}, \Gamma_{2}=\Gamma_{2}^{A} \cup \Gamma_{2}^{E}$, and by overlaying them within $\Gamma_{0}$, we see that $\Gamma_{0}$ is partitioned into at most three subarcs, each being the intersection (within $\Gamma_{0}$ ) of one of $\Gamma_{1}^{E}, \Gamma_{1}^{A}$ with one of $\Gamma_{2}^{A}, \Gamma_{2}^{E}$. See Fig. 9 (and also Fig. 7 , where the overlap has only two subarcs, of $\Gamma_{1}^{E} \cap \Gamma_{2}^{E}$ and $\Gamma_{1}^{A} \cap \Gamma_{2}^{E}$ ). Since $E_{1}$ and $E_{2}$ are disjoint, and since, by assumption, no $E$-edge of any rectangle intersects the $A$-edge of the other rectangle, the intersection $\rho_{1}(\theta)=\rho_{2}(\theta)$ can only occur within $\Gamma_{1}^{A} \cap \Gamma_{2}^{A}$. As we trace $\Gamma_{0}$ from $\alpha$ to $\beta$, we start with $\rho_{2}>\rho_{1}$, so this still holds as we reach $\Gamma_{1}^{A} \cap \Gamma_{2}^{A}$. However, the bimodality of $\rho_{1}, \rho_{2}$ and the different orientations of $Q_{1}, Q_{2}$ mean that $\rho_{1}$ is decreasing on $\Gamma_{1}^{A}$, whereas $\rho_{2}$ is increasing on $\Gamma_{2}^{A}$, so no intersection of these functions can occur within $\Gamma_{1}^{A} \cap \Gamma_{2}^{A}$, a contradiction that shows that an AA-overlap is impossible.

EE-overlap We follow the same notations as in the analysis of AA-overlaps, but use different arguments, which bring to bear the complementary rectangles $Q_{1}^{\prime}, Q_{2}^{\prime}$.

Consider the clockwise endpoint $\alpha$ of $\Gamma_{0}$, which, by construction, is the E-endpoint of $\Gamma_{1}$, incident to $\Gamma_{1}^{E}$. Consider first the subcase where $\rho_{1}(\alpha)>\rho_{2}(\alpha)$. That is, the edge $A_{1}^{*}$ of $Q_{1}$ connecting $v$ to $E_{1}$ crosses and exits $Q_{2}$ before reaching $E_{1}$; it may exit $Q_{2}$ either at $E_{2}$ (as depicted in Fig. 8(ii)) or at $A_{2}$ (as depicted in Fig. 10).

If $A_{1}^{*}$ exits $Q_{2}$ at $E_{2}$ then we follow $E_{2}$ into the complementary rectangle $Q_{1}^{\prime}$. By our assumption (that no intersection as stated in the lemma occurs) $E_{2}$ cannot exit $Q_{1}^{\prime}$ through its anchor side $A_{1}^{\prime}$ (as depicted in Fig. 11(i)). So $E_{2}$ must end inside $Q_{1}^{\prime}$, at an endpoint $q_{2}$ (see Fig. 11(ii)). But then the right angle $q_{2} w_{2} v$ must either cross the anchor $A_{1}^{\prime}$ twice, or be fully contained in $Q_{1}^{\prime}$. In the latter case $w_{2}$ lies in $Q_{1}^{\prime} \subset R_{1}$, contrary to the assumption that $v$ is non-terminal, and in the former case $w_{2}$ lies in the disk with $A_{1}^{\prime}$ as a diameter, which is also contained in $K_{1}$, and again we have a contradiction. 


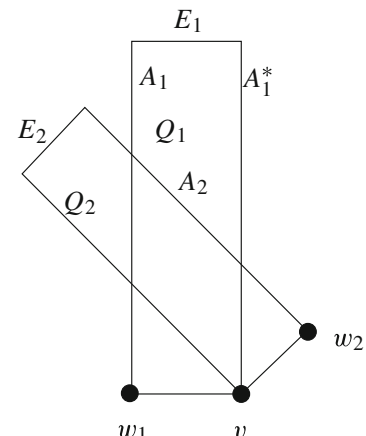

(i)

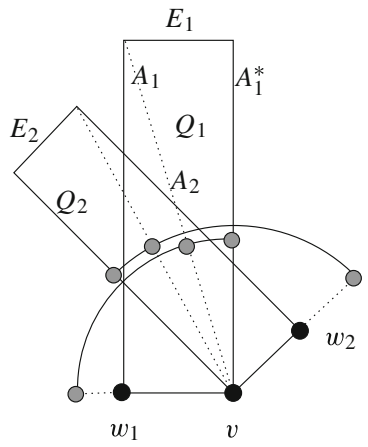

(ii)

Fig. 10 (i) Another instance of an EE-overlap. (ii) The intersection of the viewing arcs; it consists of three subarcs (in counterclockwise order): $\Gamma_{1}^{E} \cap \Gamma_{2}^{A}, \Gamma_{1}^{A} \cap \Gamma_{2}^{A}$, and $\Gamma_{1}^{A} \cap \Gamma_{2}^{E}$. Here $w_{2}$ lies in the diametral disk (not drawn) spanned by the edge $A_{1}^{*}$ of $Q_{1}$

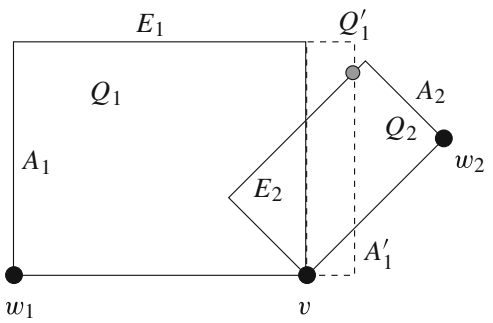

(i)

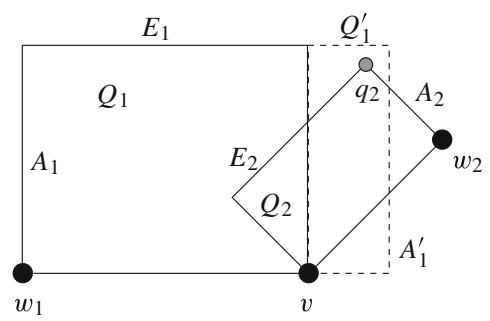

(ii)

Fig. 11 Another instance of an EE-overlap. The intersection of the viewing arcs consists of just $\Gamma_{1}^{E} \cap \Gamma_{2}^{E}$. (i) $E_{2}$ crosses the anchor $A_{1}^{\prime}$ of the complementary rectangle $Q_{1}^{\prime}$. (ii) $E_{2}$ ends inside $Q_{1}^{\prime}$

If $A_{1}^{*}$ exits $Q_{2}$ at $A_{2}$, the argument is simpler, because then $w_{2}$ is contained in the disk with $A_{1}^{*}$ as a diameter, which is contained in $K_{1}$, again contrary to the assumption that $v$ is non-terminal (see Fig. 10).

A fully symmetric argument leads to a contradiction in the case where $\rho_{1}(\beta)<$ $\rho_{2}(\beta)$. It therefore remains to consider the case where $\rho_{1}(\alpha)<\rho_{2}(\alpha)$ and $\rho_{1}(\beta)>$ $\rho_{2}(\beta)$. Here we argue exactly as in the case of AA-overlaps, using the bimodality of $\rho_{1}$ and $\rho_{2}$, that this case cannot happen. (Fig. 9 depicts the situation in this case too.) Specifically, there has to exist an intersection point of $\rho_{1}$ and $\rho_{2}$ within $\Gamma_{0}$, and it can only occur at $\Gamma_{1}^{A} \cap \Gamma_{2}^{A}$. But over this subarc $\rho_{1}$ is decreasing and $\rho_{2}$ is increasing, and we enter this subarc with $\rho_{1}<\rho_{2}$, so these functions cannot intersect within this arc. This completes the argument showing that our assumption implies that an EE-overlap is not possible.

We conclude that one of the intersections stated in the lemma must exist.

The charging scheme We charge $v$ to a portal $\left(A_{2}\right.$ or $\left.A_{2}^{\prime}\right)$ of $R_{2}$ that intersects $E_{1}$ or to a portal ( $A_{1}$ or $A_{1}^{\prime}$ ) of $R_{1}$ that intersects $E_{2}$. At least one such intersection must exist by Lemma 2 . A useful property of this charging, which will be needed in the next part of the analysis, is given by the following lemma. 
Fig. 12 Illustrating the proof of Lemma 3. Only the lower portions of $R_{i}$ and $R_{j}$ are shown

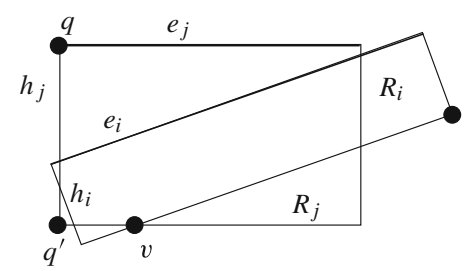

Fig. 13 Segments in $\mathcal{E}_{g}$ and the union $\mathcal{U}_{g}$ that they induce on $e_{0}^{-}$

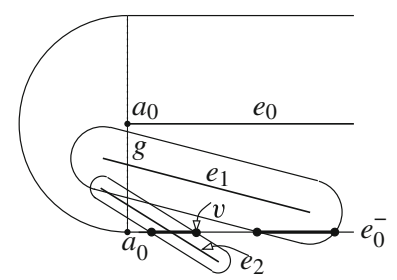

Lemma 3 Let $v$ be a non-terminal RR-vertex, lying on $\partial R_{i} \cap \partial R_{j}$, which is charged to a portal $h_{j}$ of $R_{j}$. Then $e_{i}$, traced from its intersection with $h_{j}$ into $R_{j}$, gets further away from $e_{j}$ (that is, the distance from the traced point to $e_{j}$ keeps increasing).

Proof Suppose to the contrary that $e_{i}$ approaches $e_{j}$ and assume, without loss of generality, that $e_{j}$ is horizontal, that $v$ lies on $e_{j}^{-}$, and that $h_{j}$ is the left-lower portal of $R_{j}$. In this case $e_{i}$ has positive slope. See Fig. 12.

Let $q$ denote the endpoint of $h_{j}$ incident to $e_{j}$ and let $q^{\prime}$ denote the lower endpoint of $h_{j}$. (Note that $q^{\prime}$ is $w_{j}$ if $v$ is charged to the portal $A_{j}$ of $R_{j}$ which is also a portal of $Q_{j}$ and $q^{\prime}$ is the endpoint of $A_{j}^{\prime}$, the portal of the complementary rectangle $Q_{j}^{\prime}$, otherwise.) Since $v$ is a non-terminal RR-vertex, the segment $\overrightarrow{v q^{\prime}}$, as we trace it from $v$, enters $R_{i}$ (this follows as $e_{i}$ has positive slope) and then exits it before reaching $q^{\prime}$. The exit point lies on a portal $h_{i}$ of $R_{i}$. Since $e_{i}$ intersects $h_{j}$, it follows that $h_{i}$ must also cross $h_{j}$. But then $q^{\prime}$ must lie inside the diametral disk spanned by $h_{i}$, and thus it lies inside $K_{i}$, a contradiction (to $v$ being non-terminal) that completes the proof.

The expected number of vertices charged to a portal Fix a segment of $\mathcal{E}$, denote it as $e_{0}$, and rename the other segments as $e_{1}, \ldots, e_{n-1}$. Assume, for simplicity, that $e_{0}$ is horizontal. We bound the expected number of vertices charged to the lower-left portal, denoted by $g$, of the rectangle $R_{0}$ (which is incident to the left endpoint, $a_{0}$, of $e_{0}$ ); symmetric arguments will apply to the other three portals of $R_{0}$. Given a specific permutation $\left(r_{0}, \ldots, r_{n-1}\right)$ of the input set of distances $\Theta$, let $\chi_{\mathrm{RR}}\left(g ; r_{0}, \ldots, r_{n-1}\right)$ denote the number of vertices charged to $g$ if $e_{i}$ is expanded by $r_{i}$, for $i=0, \ldots, n-1$. We wish to bound $\bar{\chi}_{\mathrm{RR}}(g)$, the expected value of $\chi_{\mathrm{RR}}\left(g ; r_{0}, \ldots, r_{n-1}\right)$ with respect to the random choice of the $r_{i}$ 's, as effected by randomly shuffling them (by a random permutation acting on $\Theta$ ).

We first fix a value $r$ (one of the values $\theta_{i} \in \Theta$ ) of $r_{0}$ and bound $\bar{\chi}_{\mathrm{RR}}(g \mid r)$, the expected number of vertices charged to $g$ conditioned on the choice $r_{0}=r$; the expectation is taken over those permutations that fix $r_{0}=r$; they can be regarded 
Fig. 14 Segments in $\mathcal{E}_{g}$ and the points that they induce on $\ell_{0}^{-}$

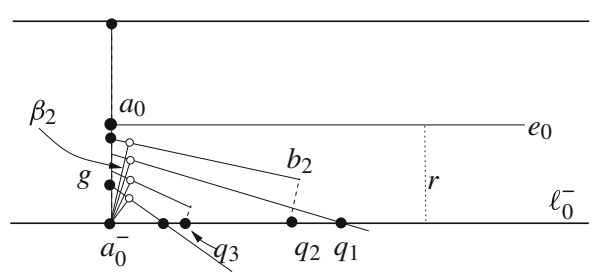

as random permutations of the remaining elements of $\Theta$. Then we bound $\bar{\chi}_{R R}(g)$ by taking the expection of the resulting bound over the choice of $r_{0}$.

So fix $r_{0}=r$. Set $K_{0}=e_{0} \oplus D(r)$, and let $\ell_{0}^{-}$denote the line supporting $e_{0}^{-}$. We have $g=a_{0} a_{0}^{-}$, and observe that all these objects depend only on $r_{0}$, so they are now fixed. By our charging scheme, if a vertex $v \in \partial R_{0} \cap \partial R_{j}$ is charged to the portal $g$, then $v \in e_{0}^{-}$, and $e_{j}$ intersects $g$. Furthermore, by Lemma 3, the slope of $e_{j}$ is negative. Let $\mathcal{E}_{g} \subseteq \mathcal{E} \backslash\left\{e_{0}\right\}$ be the set of segments that intersect $g$ and have negative slopes; the set $\mathcal{E}_{g}$ depends on the choice of $r_{0}=r$ but not on (the shuffled distances) $r_{1}, \ldots, r_{n-1}$. See Fig. 13 .

For a fixed permutation $\left(r_{1}, \ldots, r_{n-1}\right)$, set $K_{l}:=e_{l} \oplus D\left(r_{l}\right)$, for $e_{l} \in \mathcal{E}_{g}, \mathcal{K}_{g}=$ $\left\{K_{l} \mid e_{l} \in \mathcal{E}_{g}\right\}$, and $\mathcal{U}_{g}=\mathcal{U}\left(\mathcal{K}_{g}\right) \cap e_{0}^{-}$. We call a vertex of $\mathcal{U}_{g}$ an $R$-vertex if it lies on $\partial R_{i}$ for some $e_{i} \in \mathcal{E}_{g}$ (as opposed to lying on some semicircular arc). If a non-terminal RR-vertex $v$ is charged to the portal $g$, then $v$ is an R-vertex of $\mathcal{U}_{g}$ (for the specific choice $r_{0}=r$ ). It thus suffices to bound the expected number of R-vertices on $\mathcal{U}_{g}$, where the expectation is taken over the random shuffles of $r_{1}, \ldots, r_{n-1}$.

Consider a segment $e_{i} \in \mathcal{E}_{g}$. If $\ell_{0}^{-} \cap e_{i} \neq \emptyset$ then we put $q_{i}=\ell_{0}^{-} \cap e_{i}$. If $\ell_{0}^{-} \cap e_{i}=\emptyset$, then let $\lambda_{i}$ denote the line perpendicular to $e_{i}$ through $b_{i}$ (the right endpoint of $e_{i}$ ), and define $q_{i}$ to be the intersection of $\lambda_{i}$ with $\ell_{0}^{-}$. (We may assume that $q_{i}$ lies to the right of $a_{0}^{-}$, for otherwise no expansion of $e_{i}$ will be such that $R_{i}$ intersects the edge $e_{0}^{-}$.) Define $r_{i}^{*}=0$ if $\ell_{0}^{-} \cap e_{i} \neq \varnothing$ and $r_{i}^{*}=\left|b_{i} q_{i}\right|$ otherwise. For simplicity, write $\mathcal{E}_{g}$ as $\left\langle e_{1}, \ldots, e_{m}\right\rangle$, for some $m<n$, ordered so that $q_{1}, \ldots, q_{m}$ appear on $\ell_{0}^{-}$from right to left in this order; see Fig. 14. We remark that $q_{1}, \ldots, q_{m}$ are independent of the values of $r_{1}, \ldots, r_{m}$, and that the order $e_{1}, \ldots, e_{m}$ may be different from the order of the intercepts of these segments along $g$ (e.g., see the segments $e_{1}$ and $e_{2}$ in Fig. 14).

For $i=1, \ldots, m$, let $r_{i}$ be, as above, the (random) expansion distance chosen for $e_{i}$, and set $J_{i}=R_{i} \cap \ell_{0}^{-}$. If $r_{i} \leq r_{i}^{*}$ then $J_{i}=\emptyset$, and if $r_{i}>r_{i}^{*}$ then $J_{i}$ is an interval containing $q_{i}$. Let $\mathcal{U}_{0}$ be the union of the intervals $J_{i}$, and let $\mu\left(r ; r_{1}, \ldots r_{m}\right)$ be the number of connected components of $\mathcal{U}_{0}$. Clearly, each R-vertex of $\mathcal{U}_{g}$ is an endpoint of a component of $\mathcal{U}_{0}$, which implies that $\chi_{\mathrm{RR}}\left(g ; r, r_{1}, \ldots, r_{n-1}\right) \leq 2 \mu\left(r ; r_{1}, \ldots, r_{m}\right)$. It therefore suffices to bound $\bar{\mu}(r)$, the expected value of $\mu\left(r ; r_{1}, \ldots, r_{m}\right)$ over the random shuffles of $r_{1}, \ldots, r_{m}$.

For each $e_{i} \in \mathcal{E}_{g}$, let $\beta_{i}$ be the length of the segment connecting $a_{0}^{-}$to its orthogonal projection on $e_{i}$. As is easily checked, we have $\beta_{i}<r$. It is also clear that if $r_{i} \geq \beta_{i}$ then the entire segment $q_{i} a_{0}^{-}$is contained in $K_{i}$.

Lemma 4 In the preceding notations, the expected value of $\bar{\mu}(r)$ is $O(\log n)$.

Proof Assume that $r=\theta_{n-k+1}$, for some $k \in\{1, \ldots, n\}$. We claim that is this case $\bar{\mu}(r) \leq n / k$. 
For $i=1, \ldots, m$, if $r_{i}>r$ then $r_{i}>\beta_{i}$ and therefore $a_{0}^{-} \in J_{i}$. Hence, if $i$ is the smallest index for which $r_{i}>r$ (assuming that such an index exists), then $\mathcal{U}_{0}$ has at most $i$ connected components: the one containing $J_{i}$ and at most $i-1$ intervals to its right.

Recall that we condition the analysis on the choice of $r_{0}=r$, and that we are currently assuming that $r_{0}$ is the $k$ th largest value of $\Theta$. For this fixed value of $r_{0}$, the set $\mathcal{E}_{g}$ is fixed.

Order the segments in $\mathcal{E}_{0}:=\mathcal{E} \backslash e_{0}$ by placing first the $m$ segments of $\mathcal{E}_{g}$ in their order as defined above, and then place the remaining $n-m-1$ segments in an arbitrary order. Clearly this reshuffling of the segments does not affect the property that the expansion distances in $\Theta_{0}:=\Theta \backslash\{r\}$ that are assigned to them form a random permutation of $\Theta_{0}$.

In this context, $\bar{\mu}(r)$ is upper bounded by the expected value of the index $j$ of the first segment $e_{j}$ in $\mathcal{E}_{0}$ that gets one of the $k-1$ distances larger than $r$. In general, the two quantities are not equal, because we set $\mu\left(r ; r_{1}, \ldots, r_{m}\right)=m$ when $j$ is greater than $m$, that is, in case no segment of $\mathcal{E}_{g}$ gets a larger distance.

As is well known, the expected value of the index $j$ of the first appearance of one of $k-1$ distinguished elements in a random permutation of $n-1$ elements is $n / k$, from which our claim follows. This follows since the expected size of a set in an ordered partition of $n$ elements into $k$ sets is $n / k$, e.g., as in [14, p. 175, Problem 2]. Here we partition $n-1-(k-1)$ elements into $k$ sets, by placing the distinguished elements between them, and we are interested in the expected size of the first group, plus the distinguished element following it, that is $(n-1-(k-1)) / k+1=n / k$. Note that the case $k=1$ is special, because no index can get a larger value, but the resulting expectation, namely $n$, serves as an upper bound for $\bar{\mu}(r)$.

Since $r=\theta_{n-k+1}$ with probability $1 / n$, for every $k$, we have

$$
\mathrm{E}[\bar{\mu}(r)]=\sum_{k=1}^{n} \frac{1}{n} \cdot \bar{\mu}\left(\theta_{n-k+1}\right) \leq \frac{1}{n} \sum_{k=1}^{n} \frac{n}{k}=\sum_{k=1}^{n} \frac{1}{k}=O(\log n) .
$$

Putting it all together Lemma 4 proves that the expected number of non-terminal vertices of $\mathcal{U}$ charged to a fixed portal of some rectangle in $\mathcal{R}$ is $O(\log n)$. By Lemma 2, each non-terminal RR-vertex of $\mathcal{U}$ is charged to one of the $4 n$ portals of the rectangles in $\mathcal{R}$. Repeating this analysis for all these $4 n$ portals, the expected number of nonterminal RR-vertices in $\mathcal{U}$ is $O(n \log n)$. Adding the linear bound on the number of terminal RR-vertices, we obtain the following result.

Lemma 5 The expected number of RR-vertices of $\mathcal{U}(\mathcal{K})$ is $O(n \log n)$.

\subsection{CR-Vertices}

Next, we bound the expected number of CR-vertices of $\mathcal{U}$. Using a standard notation, we call a vertex $v \in \mathcal{U}$ lying on $\partial K_{i} \cap \partial K_{j}$ regular if $\partial K_{i}$ and $\partial K_{j}$ intersect at two points (one of which is $v$ ); otherwise $v$ is called irregular. By a result of Pach and Sharir [24], the number of regular vertices on $\partial \mathcal{U}$ is proportional to $n$ plus the number of irregular vertices on $\partial \mathcal{U}$. Since the expected number of RR- and CC-vertices on $\partial \mathcal{U}$ 
Fig. 15 Illustration of the proof of Lemma 6

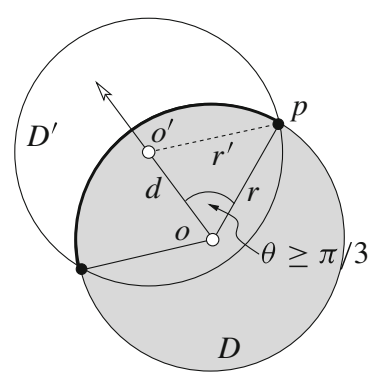

is $O(n \log n)$, the number of regular CR-vertices on $\partial \mathcal{U}$ is $O(n \log n+\kappa)$, where $\kappa$ is the number of irregular CR-vertices on $\partial \mathcal{U}$. It thus suffices to prove that the expected value of $\kappa$ is $O(n \log n)$.

Geometric properties of $C R$-vertices We begin by establishing a few simple geometric lemmas.

Lemma 6 Let $D$ and $D^{\prime}$ be two disks of respective radii $r, r^{\prime}$ and centers $o, o^{\prime}$. Assume that $r^{\prime} \geq r$ and that $o^{\prime} \in D$. Then $D^{\prime} \cap \partial D$ is an arc of angular extent at least $2 \pi / 3$, whose midpoint lies at the radius vector of $D$ from o through $o^{\prime}$.

Proof We may assume that $D$ is not fully contained in $D^{\prime}$, for otherwise the claim is trivial. Consider then the triangle $o o^{\prime} p$, where $p$ is one of the intersection points of $\partial D$ and $\partial D^{\prime}$. Put $\left|o o^{\prime}\right|=d \leq r$, and let $\angle o^{\prime} o p=\theta$; see Fig. 15. Then

$$
\cos \theta=\frac{r^{2}+d^{2}-r^{\prime 2}}{2 d r} \leq \frac{d^{2}}{2 d r}=\frac{d}{2 r} \leq \frac{1}{2} .
$$

Hence $\theta \geq \pi / 3$. Since the angular extent of $D^{\prime} \cap \partial D$ is $2 \theta$, the claim follows. The property concerning the center of the $\operatorname{arc} D^{\prime} \cap \partial D$ is also obvious.

Corollary 2 Let $D$ and $D^{\prime}$ be two disks of radii $r$ and $r^{\prime}$ and centers $o$ and $o^{\prime}$, respectively, such that $o^{\prime} \in D$. Let $D_{1}$ be some sector of $D$ of angle $\pi / 3$, and let $\gamma_{1}$ denote the circular portion of $\partial D_{1}$.

(a) If $o^{\prime} \in D_{1}$ and $r<r^{\prime}$, then $\gamma_{1}$ is fully contained in $D^{\prime}$.

(b) If $o^{\prime} \in D \backslash D_{1}$, then either $D^{\prime}$ is disjoint from $\gamma_{1}$ or $D^{\prime} \cap \gamma_{1}$ consists of one or two arcs, each containing an endpoint of $\gamma_{1}$. See Fig. 16(ii), (iii).

(In (b), no relationship between $r$ and $r^{\prime}$ is assumed.)

Proof See Fig. 16(i). Claim (a) follows from the preceding lemma, since $D^{\prime} \cap \partial D$ is an arc of angular extent at least $2 \pi / 3$ whose midpoint lies at a point on $\gamma_{1}$.

For (b), $D^{\prime} \cap \partial D$ is a connected $\operatorname{arc} \delta$, whose midpoint lies in direction $\mathbf{o o}^{\prime}$ and thus outside $\gamma_{1}$ (recall that $o^{\prime} \notin D_{1}$ ), and $D^{\prime} \cap \gamma_{1}=\delta \cap \gamma_{1}$. The intersection of two arcs of the same circle consists of zero, one, or two connected subarcs. In the first case the claim is obvious. In the third case, each of the $\operatorname{arcs} \delta, \gamma_{1}$ must contain both endpoints of the other arc, so (b) follows. In the second case, the only situation that we need to 


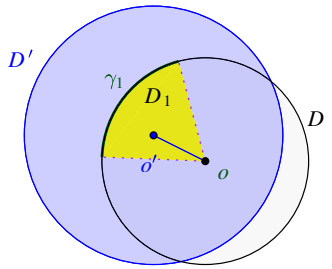

(i)

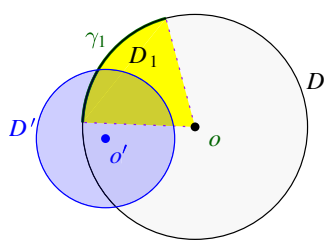

(ii)

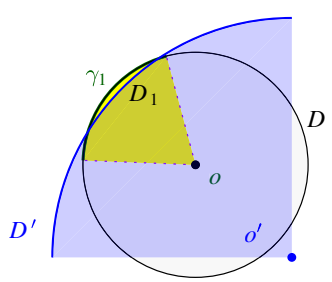

(iii)

Fig. 16 Illustration of Corollary 2: (i) the setup for (a). (ii) One possible scenario in the proof of (b), where $\delta \cap \gamma_{1}$ consists of a single arc. (iii) Another scenario in the proof of (b), where $\delta \cap \gamma_{1}$ consists of two arcs

rule out is when $\delta \cap \gamma_{1}$ is contained in the relative interior of $\gamma_{1}$, in which case $\delta$, and its midpoint, are contained in $\gamma_{1}$, contrary to assumption. Hence (b) holds in this case too.

Fix a segment of $\mathcal{E}$, call it $e_{0}$, and rename the other segments to be $e_{1}, \ldots, e_{n-1}$. $\partial K_{0}$ has two semicircular arcs, each corresponding to a different endpoint of $e_{0}$. We fix one of the semicircular arcs of $K_{0}$ and denote it by $\gamma_{0}$. Let $r_{0}$ be the random distance assigned to $e_{0}$, let $D_{0}$ be the disk of radius $r_{0}$ containing $\gamma_{0}$ on its boundary, and let $H_{0} \subset D_{0}$ be the half-disk spanned by $\gamma_{0}$.

Partition $H_{0}$ into three sectors of angular extent $\pi / 3$ each, denoted as $H_{01}, H_{02}, H_{03}$. Let $\gamma_{0 i} \subset \gamma_{0}$ denote the arc bounding $H_{0 i}$, for $i=1,2,3$. Here we call a vertex $v \in \partial U$ formed by $\gamma_{0 i} \cap \partial K_{j}$, for some $j$, a terminal vertex if the portion of $\gamma_{0 i}$ between $v$ and one of its endpoints is contained in $K_{j}$, and a non-terminal vertex otherwise. There are at most $O(1)$ terminal vertices on $\gamma_{0}$, for an overall bound of $O(n)$ on the number of such vertices, so it suffices to bound the expected number of non-terminal irregular CR-vertices on each subarc $\gamma_{0 i}$, for $i=1,2,3$.

Let $\mathcal{E}\left(r_{0}\right)$ denote the set of all segments $e_{j} \neq e_{0}$ that intersect the disk $D_{0}$, and, for $i=1,2,3$, let $\mathcal{E}_{i}\left(r_{0}\right) \subseteq \mathcal{E}\left(r_{0}\right)$ denote the set of all segments $e_{j} \neq e_{0}$ that intersect the sector $H_{0 i}$. Set $m_{i}:=m_{i}\left(r_{0}\right)=\left|\mathcal{E}_{i}\left(r_{0}\right)\right|$. Segments in $\mathcal{E}\left(r_{0}\right) \backslash \mathcal{E}_{i}\left(r_{0}\right)$ intersect $D_{0}$ but are disjoint from $H_{0 i}$. (The parameter $r_{0}$ is to remind us that all these sets depend (only) on the choice of $r_{0}$.)

Lemma 7 Let $_{j} \in \mathcal{E} \backslash \mathcal{E}\left(r_{0}\right)$. If U has a CR-vertex $v \in \gamma_{0 i} \cap \partial K_{j}$, for some $i=1,2,3$, then $v$ is either a regular vertex or a terminal vertex.

Proof Let $c$ denote the center of $D_{0}$ (it is an endpoint of $e_{0}$ ), and consider the interaction between $K_{j}$ and $D_{0}$. We split the analysis into the following two cases.

Case 1, $r_{j} \leq r_{0}$ : Regard $D_{0}$ as $D_{0}^{*} \oplus D\left(r_{j}\right)$, where $D_{0}^{*}$ is the disk of radius $r_{0}-r_{j}$ centered at $c$. By assumption, $D_{0}^{*}$ and $e_{j}$ are disjoint, implying that $D_{0}$ and $K_{j}$ are pseudo-disks (cf. [18]), that is, their boundaries intersect in two points, one of which is $v$; denote the other point as $v^{\prime}$. See Fig. 17(i).

If only $v$ lies on $\gamma_{0}$, then $v$ must be a terminal vertex, so assume that both $v$ and $v^{\prime}$ lie on $\gamma_{0}$. We claim that $\partial K_{j}$ and $\partial K_{0}$ can intersect only at $v$ and $v^{\prime}$, implying that $v$ is regular. Indeed, $v$ and $v^{\prime}$ partition $\partial K_{j}$ into two connected pieces. One piece is inside $D_{0}$, locally near $v$ and $v^{\prime}$, and cannot intersect $\partial K_{i}$ in a point other than $v$ and $v^{\prime}$ without intersecting $D_{0}$ in a third point (other than $v$ and $v^{\prime}$ ), contradicting the 


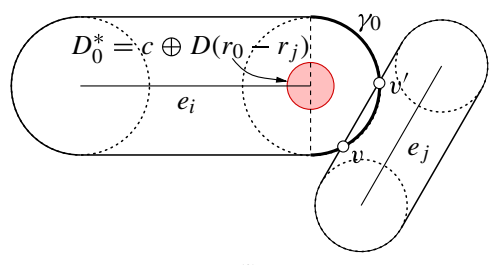

(i)

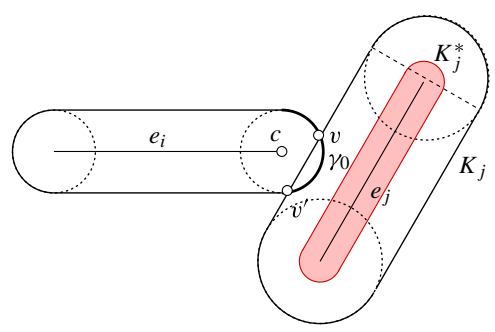

(ii)

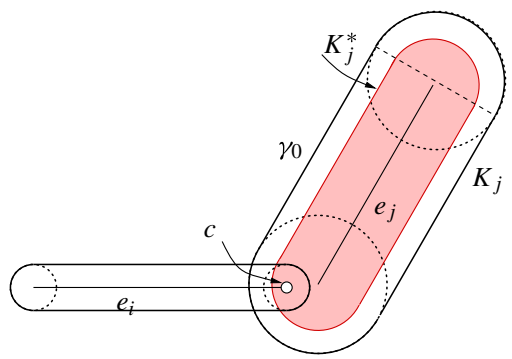

(iii)

Fig. 17 (i) The case when $r_{0} \geq r_{j}$ and $\partial K_{j} \cap \gamma_{0}$ contains the two intersection points of $\partial D_{0}$ and $\partial K_{j}$. (ii) The case when $r_{j}>r_{0}$ and $c \notin K_{j}^{*}$. (iii) The case when $r_{j}>r_{0}$ and $c \in K_{j}^{*}$

property that $D_{0}$ and $K_{j}$ are pseudo-disks. The other connected piece of $\partial K_{j}$ between $v$ and $v^{\prime}$ is separated from $\partial K_{i} \backslash \gamma_{0}$ by the line through $v$ and $v^{\prime}$ and therefore cannot contain intersections other than $v$ and $v^{\prime}$ between $\partial K_{j}$ and $\partial K_{i}$.

Case 2, $r_{j}>r_{0}$ : Let $K_{j}^{*}=e_{j} \oplus D\left(r_{j}-r_{0}\right) . K_{j}$ can now be regarded as $K_{j}^{*} \oplus D\left(r_{0}\right)$. If $c \notin K_{j}^{*}$, then by the result of [18], $D_{0}=c \oplus D\left(r_{0}\right)$ and $K_{j}$ are pseudo-disks; see Fig. 17(ii). Therefore, the argument given above for the case where $r_{0} \geq r_{j}$ implies the lemma in this case as well. Finally, $c \in K_{j}^{*}$ implies that $K_{j}$ contains $D_{0}$, so this case cannot occur (it contradicts the existence of $v$ ). See Fig. 17(iii).

Using Lemmas 6 and 7, we obtain the following property.

Lemma 8 Let $v \in \gamma_{0 i} \cap \partial K_{j}$ be a non-terminal, irregular CR-vertex of $\mathcal{U}$. Then (i) $e_{j} \in \mathcal{E}_{i}\left(r_{0}\right)$, and (ii) for all $e_{l} \in \mathcal{E}_{i}\left(r_{0}\right), r_{l}<r_{0}$.

Proof (i) Lemma 7 implies that $e_{j} \in \mathcal{E}\left(r_{0}\right)$. Suppose to the contrary that $e_{j} \in$ $\mathcal{E}\left(r_{0}\right) \backslash \mathcal{E}_{i}\left(r_{0}\right)$. Let $v^{\prime}$ be the orthogonal projection of $v$ onto $e_{j}$ ( $v^{\prime}$ exists since $v$ is a CR-vertex). Assume first that $v^{\prime}$ is inside $D_{0}$ (as in Fig. 18(i)), and consider the disk $D^{\prime}$ of radius $r_{j}$ centered at $v^{\prime}$. Clearly, $D^{\prime} \subseteq K_{j}$. Part (b) of Corollary 2 implies that $D^{\prime}$ intersects $\gamma_{0 i}$ at an arc or a pair of arcs, each containing an endpoint of $\gamma_{0 i}$, i.e., $v$ is a terminal vertex, contrary to assumption.

Assume then that $v^{\prime} \notin D_{0}$. Since $e_{j}$ intersects $D_{0}$, there exists a point $u \in e_{j} \cap \partial D_{0}$ such that $u v^{\prime}$ is openly disjoint from $D_{0}$. By our assumption that $e_{j} \in \mathcal{E}\left(r_{0}\right) \backslash \mathcal{E}_{i}\left(r_{0}\right)$, $u \notin \gamma_{0 i}$. Let $\delta$ denote the arc of $\partial D_{0}$ that connects $u$ and $v$ and lies, locally near $u$, on the side of $e_{j}$ that contains $v$. See Fig. 18(ii). It can be verified that $\delta$ is the smaller of the two arcs of $\partial D_{0}$ connecting $u$ and $v$. Clearly, $\delta$ contains an endpoint of $\gamma_{0 i}$, and the desired contradiction will be reached by showing that $\delta$ is contained in $K_{j}$. 


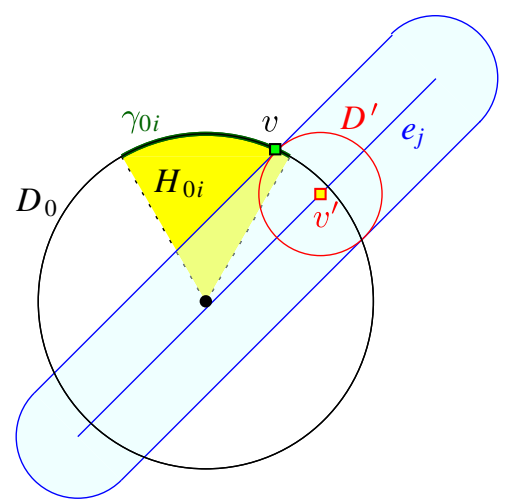

(i)

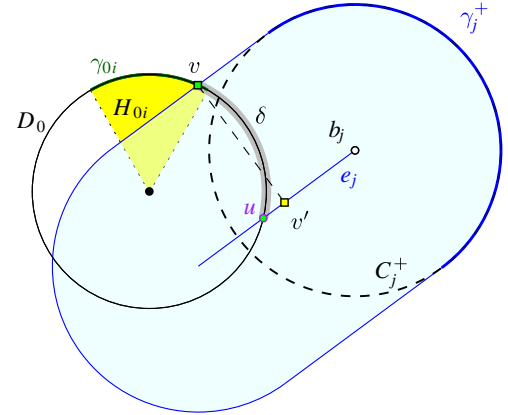

(ii)

Fig. 18 Illustration of the proof of Lemma 8: (i) the case where $v^{\prime} \in D_{0}$. (ii) The case where $v^{\prime} \notin D_{0}$

The $\operatorname{arc} \delta$ lies inside a wedge $W$, with apex $u$, formed by the ray $u v$ and the line supporting $e_{j}$, and also inside the strip $\Sigma$ bounded by $e_{j}$ and the parallel line through $v$. Let $b_{j}$ be the endpoint of $e_{j}$ that lies on the boundary of $W, \gamma_{j}^{+}$the circular arc of $K_{j}$ centered at $b_{j}$, and $C_{j}^{+}$the circle (centered at $b_{j}$ ) containing the arc $\gamma_{j}^{+}$. Since $\delta \subset W \cap \Sigma, \delta$ can intersect $\partial K_{j}$ only at a point on the arc $\gamma_{j}$. Therefore, if $\delta \not \subset K_{j}$, then $\delta$ escapes $K_{j}$ through a point $w \in \gamma_{j}^{+}$. Note that the endpoint $v$ of $\delta$ lies outside $C_{j}^{+}$, so the portion of $\delta$ between $v$ and $w$ intersects $C_{j}^{+}$at a point other than $w$. Finally, the endpoint $u$ of $\delta$ lies inside $K_{j}$, so the portion of $\delta$ between $w$ and $u$, which lies outside $D_{j}^{+}$locally near $w$, also intersects $\gamma_{j}^{+}$at a point different from $w ; \delta$ enters the disk $D_{j}^{+}$bounded by $C_{j}^{+}$at this point. This, however, implies that $\delta$ intersect $C_{j}^{+}$at three points, a contradiction. Hence $\delta \subset K_{j}$.

To establish (ii), notice that Part (a) of Corollary 2 implies that $r_{l}<r_{0}$ for all $e_{l} \in \mathcal{E}_{i}\left(r_{0}\right)$, because otherwise we would have $\gamma_{0 i} \subset K_{l}$ and $\gamma_{0 i}$ would not contain any vertex of $\partial \mathcal{U}$.

The expected number of non-terminal vertices on $\gamma_{0}$. We are now ready to bound the expected number of non-terminal irregular CR-vertices of $\mathcal{U}$ that lie on the semicircular arc $\gamma_{0}$ of $K_{0}$. Note that $\gamma_{0}$ is not fixed, as it depends on the value of $r_{0}$. Let $\chi_{\mathrm{CR}}\left(\gamma_{0} ; r_{0}, r_{1}, \ldots, r_{n-1}\right)$ denote the number of non-terminal irregular vertices on $\gamma_{0}$, assuming that $r_{i}$ is the expansion distance of $K_{i}$, for $i=0, \ldots, n-1$. Our goal is to bound

$$
\bar{\chi}_{\mathrm{CR}}\left(\gamma_{0}\right)=\mathrm{E}\left[\chi_{\mathrm{CR}}\left(\gamma_{0} ; r_{0}, \ldots, r_{n-1}\right)\right]
$$

where the expectation is over all the random permutations assigning these distances to the segments of $\mathcal{E}$. As for RR-vertices, we first fix the value of $r_{0}$ to, say, $r$, and bound $\chi_{\mathrm{CR}}\left(\gamma_{0} \mid r\right)$, the expected value of $\chi_{\mathrm{CR}}\left(\gamma_{0}, r, r_{1}, \ldots, r_{n-1}\right)$, where the expectation is taken over the random shuffles of $r_{1}, \ldots, r_{n-1}$, and then bound $\bar{\chi}_{\mathrm{CR}}\left(\gamma_{0}\right)$ by taking the expectation of the bound over the choice of $r_{0}$. 
Lemma 9 Using the notation above, $\bar{\chi}_{\mathrm{CR}}\left(\gamma_{0}\right)=O(\log n)$.

Proof Following the above scheme, suppose that the value $r_{0}$ is indeed fixed to $r$, so $\gamma_{0}$ and $\gamma_{0 i}, 1 \leq i \leq 3$, are fixed. As above, set $m_{i}=\left|\varepsilon_{i}(r)\right|$, for $i=1,2,3$; the sets $\mathcal{E}_{i}(r)$ and their sizes $m_{i}$ are also fixed. We bound the expected number of non-terminal irregular vertices on $\gamma_{0 i}$, for a fixed $i \in\{1,2,3\}$. By Lemma 8 , any such vertex lies on the boundary of $\mathcal{J}_{0}$, the intersection of $\gamma_{0 i}$ with the union of $\left\{K_{l} \mid e_{l} \in \mathcal{E}_{i}(r)\right\}$. Equivalently, it suffices to bound the expected number of connected components of $\mathcal{J}_{0}$ that lie in the interior of $\gamma_{0 i}$. By Lemma 8 , if $r_{l} \geq r$ for any $e_{l} \in \mathcal{E}_{i}(r)$, then there are no such components.

Assume that $r=\theta_{n-k+1}$ for some $k \in\{1, \ldots, n\}$. To bound $\chi_{\mathrm{CR}}\left(\gamma_{0} \mid r\right)$, we first bound the probability $p$ that all the $m_{i}$ radii that are assigned to the segments of $\mathcal{E}_{i}(r)$ are smaller than $r$. We have

$$
\begin{aligned}
p & =\frac{\left(\begin{array}{c}
n-k \\
m_{i}
\end{array}\right)}{\left(\begin{array}{c}
n-1 \\
m_{i}
\end{array}\right)}=\frac{(n-k)(n-k-1) \cdots\left(n-k-m_{i}+1\right)}{(n-1)(n-2) \cdots\left(n-m_{i}\right)} \\
& =\left(1-\frac{k-1}{n-1}\right)\left(1-\frac{k-1}{n-2}\right) \cdots\left(1-\frac{k-1}{n-m_{i}}\right) \\
& <\left(1-\frac{k-1}{n-1}\right)^{m_{i}}<\mathrm{e}^{-(k-1) m_{i} /(n-1)}
\end{aligned}
$$

By Lemma 8 (ii), with probability $1-p$, there are no connected components. In the complementary case, when all the $m_{i}$ radii under consideration are smaller than $r$, we pessimistically bound the number of connected components by $2 m_{i}$ - each segment of $\mathcal{E}_{i}(r)$ can generate at most two connected components. In other words, when $k \geq 2$, the expected number of connected components of $\mathcal{\partial}_{0}$ is at most

$$
\begin{aligned}
2 m_{i} p & <2 m_{i} \mathrm{e}^{-(k-1) m_{i} /(n-1)} \\
& =\frac{2(n-1)}{k-1} \cdot\left(\left((k-1) m_{i} /(n-1)\right) \mathrm{e}^{-(k-1) m_{i} /(n-1)}\right) \\
& <\frac{2(n-1)}{\mathrm{e}(k-1)}
\end{aligned}
$$

because the maximum value of the expression $x e^{-x}$ is $e^{-1}$. For $k=1$, the value of $p$ is $q$, so the expected number of connected components in this case is $2 m_{i} \leq 2(n-1)$.

Since $r=\theta_{n-k+1}$ with probability $1 / n$ for every $k$, we have

$$
\begin{aligned}
\mathrm{E}\left[\bar{\chi}_{\mathrm{CR}}\left(\gamma_{0}\right)\right] & =\mathrm{E}\left[\chi_{\mathrm{CR}}\left(\gamma_{0} \mid r\right)\right]=\sum_{k=1}^{n} \frac{1}{n} \cdot \mathrm{E}\left[\chi_{\mathrm{CR}}\left(\gamma_{0} \mid \theta_{n-k+1}\right)\right] \\
& \leq \frac{1}{n}\left[2(n-1)+\sum_{k=2}^{n} \frac{2(n-1)}{e(k-1)}\right] \\
& =O\left(\sum_{k=1}^{n} \frac{1}{k}\right)=O(\log n) .
\end{aligned}
$$


Summing this bound over all three subarcs of $\gamma_{0}$ and adding the constant bound on the number of terminal (irregular) vertices, we obtain that the expected number of irregular CR-vertices of $\mathcal{U}$ on $\gamma_{0}$ is $O(\log n)$. Summing these expectations over the $2 n$ semicircular arcs of the racetracks in $\mathcal{K}$, and adding the bounds on the number of regular CR-vertices we obtain the following lemma.

Lemma 10 The expected number of $C R$-vertices on $\mathcal{U}(\mathcal{K})$ is $O(n \log n)$.

Combining Lemma 5, Lemma 10, and the linear bound on the number of CCvertices, completes the proof of Theorem 2 for the case of segments.

\section{The Case of Polygons}

In this section we consider the case where the objects of $\mathcal{C}$ are $n$ convex polygons, each with at most $s$ vertices. For simplicity, we prove Theorem 2 when each $C_{i}$ is a convex $s$-gon-if $C_{i}$ has fewer than $s$ vertices, we can split some of its edges into multiple edges so that it has exactly $s$ vertices. We reduce this case to the case of segments treated above. A straightforward reduction that just takes the individual edges of the $s$-gons as our set of segments does not work, since edges of the same polygon are all expanded by the same distance. Nevertheless, we can overcome this difficulty as follows.

Let $\mathcal{C}=\left\{C_{1}, \ldots, C_{n}\right\}$ be the set of the $n$ given polygons, and consider a fixed assignment of expansion distances $r_{i}$ to the polygons $C_{i}$. For each $i$, enumerate the edges of $C_{i}$ as $e_{i 1}, e_{i 2}, \ldots, e_{i s}$; the order of enumeration is not important. Let $v$ be a vertex of $\partial \mathcal{U}$, lying on the boundaries of $K_{i}$ and $K_{j}$, for some $1 \leq i<j \leq n$. Then there exist an edge $e_{i p}$ of $C_{i}$ and an edge $e_{j q}$ of $C_{j}$ such that $v$ lies on $\partial\left(e_{i p} \oplus D\left(r_{i}\right)\right)$ and on $\partial\left(e_{j q} \oplus D\left(r_{j}\right)\right)$; the choice of $e_{i p}$ is unique if the portion of $\partial K_{i}$ containing $v$ is a straight edge, and, when that portion is a circular arc, any of the two edges incident to the center of the corresponding disk can be taken to be $e_{i p}$. A similar property holds for $e_{j q}$.

The following stronger property holds too. For each $1 \leq p \leq s$, let $\mathcal{C}_{p}$ be the set of edges $\left\{e_{1 p}, e_{2 p}, \ldots, e_{n p}\right\}$, and let $\mathcal{K}_{p}=\left\{e_{1 p} \oplus D\left(r_{1}\right), \ldots, e_{n p} \oplus D\left(r_{n}\right)\right\}$. Then, as is easily verified, $v$ is a vertex of the union $\mathcal{U}\left(\mathcal{K}_{p} \cup \mathcal{K}_{q}\right)$. Moreover, for each $p$, the expansion distances $r_{i}$ of the edges $e_{i p}$ of $\mathcal{C}_{p}$ are all the elements of $\Theta$, each appearing once, and their assignment to the segments of $\mathcal{C}_{p}$ is a random permutation. Fix a pair of indices $1 \leq p<q \leq s$, and note that each expansion distance $r_{i}$ is assigned to exactly two segments of $\mathcal{C}_{p} \cup \mathcal{C}_{q}$, namely, to $e_{i p}$ and $e_{i q}$.

We now repeat the analysis given in the preceding section for the collection $\mathcal{C}_{p} \cup \mathcal{C}_{q}$, and make the following observations. First, the analysis of $\mathrm{CC}$-vertices remains the same, since the complexity of the union of any family of disks is linear.

Second, in the analysis of RR- and CR-vertices, the exploitation of the random nature of the distances $r_{i}$ comes into play only after we have fixed one segment (that we call $\left.e_{0}\right)$ and its expansion distance $r_{0}$, and consider the expected number of RRvertices and CR-vertices on the boundary of $K_{0}=e_{0} \oplus D\left(r_{0}\right)$, conditioned on the fixed choice of $r_{0}$. Suppose, without loss of generality, that $e_{0}$ belongs to $\mathcal{C}_{p}$. We first ignore its sibling $e_{0}^{\prime}$ in $\mathrm{C}_{q}$ (from the same polygon), which receives the same expansion 
distance $r_{0} ; e_{0}^{\prime}$ can form only $O(1)$ vertices of $\mathcal{U}$ with $e_{0} .{ }^{2}$ The interaction of $e_{0}$ with the other segments of $\mathcal{C}_{p}$ behaves exactly as in Sect. 3 , and yields an expected number of $O(\log n)$ RR-vertices of $\mathcal{U}\left(\mathcal{K}_{p}\right)$ charged to the portals of $R_{0}$ and an expected number of $O(\log n) \mathrm{CR}$-vertices charged to circular arcs of $K_{0}$. Similarly, the interaction of $e_{0}$ with the other segments of $\mathcal{C}_{q}$ (excluding $e_{0}^{\prime}$ ) is also identical to that in Sect. 3 , and yields an additional expected number of $O(\log n)$ vertices of $\mathcal{U}\left(\left\{e_{0}\right\} \cup \mathcal{K}_{q}\right)$ charged to an portals and circular arcs of $K_{0}$. Since any vertex of $\mathcal{U}\left(\mathcal{K}_{p} \cup \mathcal{K}_{q}\right)$ involving $e_{0}$ must be one of these two kinds of vertices, we obtain a bound of $O(\log n)$ on the expected number of such vertices, and summing this bound over all segments $e_{0}$ of $\mathcal{C}_{p} \cup \mathcal{C}_{q}$, we conclude that the expected complexity of $\mathcal{U}\left(\mathcal{K}_{p} \cup \mathcal{K}_{q}\right)$ is $O(n \log n)$. Note also that the analysis just given manages to finesse the issue of segments sharing endpoints.

Summing this bound over all $O\left(s^{2}\right)$ choices of $p$ and $q$, we obtain the bound asserted in Theorem 2. The constant of proportionality in the bound that this analysis yields is $O\left(s^{2}\right)$.

\section{Network Vulnerability Analysis}

Let $\mathcal{E}=\left\{e_{1}, \ldots, e_{n}\right\}$ be a set of $n$ segments in the plane with pairwise-disjoint relative interiors, and let $\varphi: \mathbb{R}_{\geq 0} \rightarrow[0,1]$ be an edge failure probability function such that $1-\varphi$ is a cdf. For each segment $e_{i}$, define the function $f_{i}: \mathbb{R}^{2} \rightarrow[0,1]$ by $f_{i}(q)=\varphi\left(d\left(q, e_{i}\right)\right)$, for $q \in \mathbb{R}^{2}$, where $d\left(q, e_{i}\right)$ is the distance from $q$ to $e_{i}$, and set

$$
\Phi(q, \mathcal{E})=\sum_{i=1}^{n} f_{i}(q) .
$$

In this section we present a Monte-Carlo algorithm, which is an adaptation and a simplification of the algorithm described in [2], for computing a location $\tilde{q}$ such that $\Phi(\tilde{q}, \mathcal{E}) \geq(1-\delta) \Phi(\mathcal{E})$, where $0<\delta<1$ is some prespecified error parameter, and where

$$
\Phi(\mathcal{E})=\max _{q \in \mathbb{R}^{2}} \Phi(q, \mathcal{E})
$$

The expected running time of the algorithm is a considerable improvement over the algorithm in [2]; this improvement is a consequence of the bounds obtained in the preceding sections.

To obtain the algorithm we first discretize each $f_{i}$ by choosing a finite family $\mathcal{K}_{i}$ of super-level sets of $f_{i}$ (each of the form $\left\{q \in \mathbb{R}^{2} \mid f_{i}(q) \geq t\right\}$ ), and reduce the problem of computing $\Phi(\mathcal{E})$ to that of computing the maximum depth in the arrangement $\mathcal{A}(\mathcal{K})$ of $\mathcal{K}=\bigcup_{i} \mathcal{K}_{i}$. Our algorithm then uses a sampling-based method for estimating the maximum depth in $\mathcal{A}(\mathcal{K})$, and thereby avoids the need to construct $\mathcal{A}(\mathcal{K})$ explicitly.

\footnotetext{
2 As a matter of fact, $e_{0}$ and $e_{0}^{\prime}$ do not generate any vertex of the full union $\mathcal{U}(\mathcal{C})$, but they might generate vertices of the partial union $\mathcal{U}\left(\mathcal{C}_{p} \cup \mathcal{C}_{q}\right)$.
} 


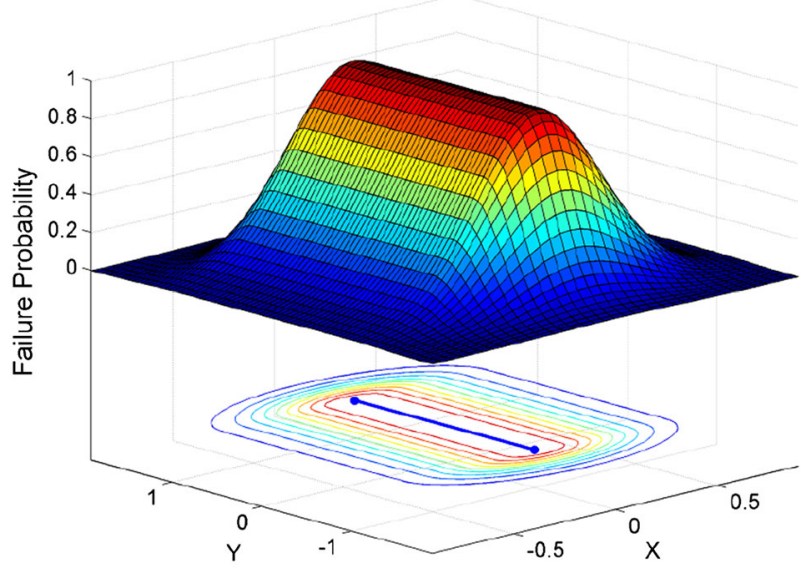

Fig. 19 Discretizing the function $f_{e}$ for an edge $e$

In more detail, set $m=\lceil 2 n / \delta\rceil$. For each $1 \leq j<m$, let $r_{j}=\varphi^{-1}(1-j / m)$, and let, for $i=1, \ldots, n, K_{i j}=e_{i} \oplus D\left(r_{j}\right)$ be the racetrack formed by the Minkowski sum of $e_{i}$ with the disk of radius $r_{j}$ centered at the origin. Note that $r_{j}$ increases with $j$. Set $\tilde{\varphi}=\left\{r_{j} \mid 1 \leq j<m\right\}, \mathcal{K}_{i}=\left\{K_{i j} \mid 1 \leq j<m\right\}$, and $\mathcal{K}=\bigcup_{1 \leq i \leq n} \mathcal{K}_{i}$. See Fig. 19. Note that we cannot afford to, and indeed do not, compute $\mathcal{K}$ explicitly, as its cardinality (which is quadratic in $n$ ) is too large.

For a point $q \in \mathbb{R}^{2}$ and for a subset $\mathrm{X} \subseteq \mathcal{K}$, let $\Delta(q, \mathrm{X})$, the depth of $q$ with respect to $\mathrm{X}$, be the number of racetracks of $\mathrm{X}$ that contain $q$ in their interior, and let

$$
\Delta(\mathrm{X})=\max _{q \in \mathbb{R}^{2}} \Delta(q, \mathrm{X})
$$

The following lemma (whose proof, which is straightforward, can be found in [2]) shows that the maximum depth of $\mathcal{K}$ approximates $\Phi(\mathcal{E})$.

Lemma 11 (Agarwal et al. [2])

(i) $\Phi(q, \mathcal{E}) \geq \frac{\Delta(q, \mathcal{K})}{m}$, for each point $q \in \mathbb{R}^{2}$.

(ii) $\Phi(\mathcal{E}) \geq \frac{\Delta(\mathcal{K})}{m} \geq\left(1-\frac{1}{2} \delta\right) \Phi(\mathcal{E})$.

By Lemma 11 , it suffices to compute a point $\tilde{q}$ of depth at least $\left(1-\frac{1}{2} \delta\right) \Delta(\mathcal{K})$ in $\mathcal{A}(\mathcal{K})$; by (i) and (ii) we will then have

$$
\Phi(\tilde{q}, \mathcal{E}) \geq \frac{\Delta(\tilde{q}, \mathcal{K})}{m} \geq\left(1-\frac{1}{2} \delta\right) \frac{\Delta(\mathcal{K})}{m} \geq\left(1-\frac{1}{2} \delta\right)^{2} \Phi(\mathcal{E})>(1-\delta) \Phi(\mathcal{E}) .
$$

We describe a Monte-Carlo algorithm for computing such a point $\tilde{q}$, which is a simpler variant of the algorithm described in [6] (see also [4]), but we first need the following definitions. 
For a point $q \in \mathbb{R}^{2}$ and for a subset $\mathrm{X} \subseteq \mathcal{K}$, let $\omega(q, \mathrm{X})=\frac{\Delta(q, \mathrm{X})}{|\mathrm{X}|}$ be the fractional depth of $q$ with respect to $\mathrm{X}$, and let

$$
\omega(\mathrm{X})=\max _{q \in \mathbb{R}^{2}} \omega(q, \mathrm{X})=\frac{\Delta(\mathrm{X})}{|\mathrm{X}|}
$$

We observe that $\Delta(\mathcal{K}) \geq m-1$ because the depth near each $e_{i}$ is at least $m-1$. Hence,

$$
\omega(\mathcal{K}) \geq \frac{m-1}{|\mathcal{K}|}=\frac{m-1}{(m-1) n}=\frac{1}{n} .
$$

Our algorithm estimates fractional depths of samples of $\mathcal{K}$ and computes a point $\tilde{q}$ such that $\omega(\tilde{q}, \mathcal{K}) \geq\left(1-\frac{1}{2} \delta\right) \omega(\mathcal{K})$. By definition, this is equivalent to $\Delta(\tilde{q}, \mathcal{K}) \geq$ $\left(1-\frac{1}{2} \delta\right) \Delta(\mathcal{K})$, which is what we need.

We also need the following concept from the theory of random sampling.

For two parameters $0<\rho, \varepsilon<1$, we call a subset $\mathrm{A} \subseteq \mathcal{K}$ a $(\rho, \varepsilon)$-approximation if the following holds for all $q \in \mathbb{R}^{2}$ :

$$
|\omega(q, \mathcal{K})-\omega(q, \mathrm{~A})| \leq \begin{cases}\varepsilon \omega(q, \mathcal{K}) & \text { if } \omega(q, \mathcal{K}) \geq \rho \\ \varepsilon \rho & \text { if } \omega(q, \mathcal{K})<\rho\end{cases}
$$

This notion of $(\rho, \varepsilon)$-approximation is a special case of the notion of relative $(\rho, \varepsilon)$ approximation defined in [16] for general range spaces with finite VC-dimension. The special case at hand applies to the so-called dual range space $\left(\mathcal{K}, \mathbb{R}^{2}\right)$, where the ground set $\mathcal{K}$ is our collection of racetracks, and where each point $q \in \mathbb{R}^{2}$ defines a range equal to the set of racetracks containing $q$; here $\Delta(q, \mathcal{K})$ is the size of the range defined by $q$, and $\omega(q, \mathcal{K})$ is its relative size. Since $\left(\mathcal{K}, \mathbb{R}^{2}\right)$ has finite VC-dimension (see, e.g., [25]), it follows from a result in [16] that, for any integer $b$, a random subset of size

$$
\nu(\rho, \varepsilon):=\frac{c b}{\varepsilon^{2} \rho} \ln n
$$

is a $(\rho, \varepsilon)$-approximation of $\mathcal{K}$ with probability at least $1-1 / n^{b}$, where $c$ is a sufficiently large constant (proportional to the VC-dimension of our range space). In what follows we fix $b$ to be a sufficiently large integer, so as to guarantee (via the probability union bound) that, with high probability, all the samples that we construct in the algorithm will have the desired approximation property.

The algorithm works in two phases. The first phase finds a value $\rho \geq 1 / n$ such that $\omega(\mathcal{K}) \in[\rho, 2 \rho]$. The second phase exploits this "localization" of $\omega(\mathcal{K})$ to compute the desired point $\tilde{q}$.

The first phase performs a decreasing exponential search: For $i \geq 1$, the $i$ th step of the search tests whether $\omega(\mathcal{K}) \leq 1 / 2^{i}$. If the answer is YES, the algorithm moves to the $(i+1)$-st step; otherwise it switches to the second phase. Since we always have $\omega(\mathcal{K}) \geq 1 / n$ (see (2)), the first phase consists of at most $\left\lceil\log _{2} n\right\rceil$ steps. 
At the $i$ th step of the first phase, we fix the parameters $\rho_{i}=1 / 2^{i}$ and $\varepsilon=1 / 8$, and construct a $\left(2 \rho_{i}, \varepsilon\right)$-approximation of $\mathcal{K}$ by choosing a random subset $\mathcal{R}_{i} \subset \mathcal{K}$ of size $v_{i}=\nu\left(2 \rho_{i}, \varepsilon\right)=O\left(2^{i} \log n\right)$. We construct $\mathcal{A}\left(\mathcal{R}_{i}\right)$, e.g., using the randomized incremental algorithm described in [25, Chapter 4] and compute $\omega\left(\mathcal{R}_{i}\right)$ by traversing the arrangement $\mathcal{A}\left(\mathcal{R}_{i}\right)$. Then, if

$$
\omega\left(\mathcal{R}_{i}\right) \leq(1-2 \varepsilon) \rho_{i}=\frac{3}{4} \rho_{i}
$$

we continue to step $i+1$ of the first phase. Otherwise, we switch to the second phase of the algorithm (which is described below). The following lemma establishes the important properties of the first phase.

Lemma 12 When the algorithm reaches step $i$ of the first phase, we have $\omega(\mathcal{K}) \leq \rho_{i-1}$ and $\omega(\mathcal{K})$ is within the interval $\left[\omega\left(\mathcal{R}_{i}\right)-\frac{1}{4} \rho_{i}, \omega\left(\mathcal{R}_{i}\right)+\frac{1}{4} \rho_{i}\right]$.

Proof The proof is by induction on the steps of the algorithm. Assume that the algorithm is in step $i$. Then by induction $\omega(\mathcal{K}) \leq \rho_{i-1}=2 \rho_{i}$ (for $i=1$ this is trivial since $\left.\rho_{i-1}=1\right)$. This, together with $\mathcal{R}_{i}$ being a $\left(2 \rho_{i}, \varepsilon\right)$-approximation of $\mathcal{K}$, implies by (3) that

$$
\omega(\mathcal{K})=\omega\left(q^{*}, \mathcal{K}\right) \leq \omega\left(q^{*}, \mathcal{R}_{i}\right)+2 \varepsilon \rho_{i} \leq \omega\left(\mathcal{R}_{i}\right)+2 \varepsilon \rho_{i}=\omega\left(\mathcal{R}_{i}\right)+\frac{1}{4} \rho_{i},
$$

where $q^{*}$ is a point satisfying $\omega\left(q^{*}, \mathcal{K}\right)=\omega(\mathcal{K})$. Furthermore, using (3) again (in the opposite direction), we conclude that

$$
\omega(\mathcal{K}) \geq \omega\left(q_{i}, \mathcal{K}\right) \geq \omega\left(q_{i}, \mathcal{R}_{i}\right)-2 \varepsilon \rho_{i}=\omega\left(\mathcal{R}_{i}\right)-\frac{1}{4} \rho_{i},
$$

where $q_{i}$ is a point satisfying $\omega\left(q_{i}, \mathcal{R}_{i}\right)=\omega\left(\mathcal{R}_{i}\right)$. So we conclude that $\omega(\mathcal{K})$ is in the interval specified by the lemma.

The algorithm continues to step $i+1$ if $\omega\left(\mathcal{R}_{i}\right) \leq \frac{3}{4} \rho_{i}$. But then by (5) we get that $\omega(\mathcal{K}) \leq \frac{3}{4} \rho_{i}+\frac{1}{4} \rho_{i}=\rho_{i}$ as required.

Suppose that the algorithm decides to terminate the first phase and continue to the second phase, at step $i$. Then, by Lemma 4.2, we have that $\omega(\mathcal{K}) \in\left[\omega\left(\mathcal{R}_{i}\right)-\right.$ $\left.\frac{1}{4} \rho_{i}, \omega\left(\mathcal{R}_{i}\right)+\frac{1}{4} \rho_{i}\right]$. Since, by construction, $\omega\left(\mathcal{R}_{i}\right)>\frac{3}{4} \rho_{i}$, the ratio between the endpoints of this interval is at most 2 , as is easily checked, so if we set $\rho=\omega\left(\mathcal{R}_{i}\right)-\frac{1}{4} \rho_{i}$ then $\omega(\mathcal{K}) \in[\rho, 2 \rho]$ as required upon entering the second phase.

In the second phase, we set $\rho=\omega\left(\mathcal{R}_{i}\right)-\frac{1}{4} \rho_{i}$ and $\varepsilon=\delta / 4$, and construct a $(\rho, \varepsilon)$ approximation of $\mathcal{K}$ by choosing, as above, a random subset $\mathcal{R}$ of size $v=v(\rho, \varepsilon)=$ $O\left(2^{i} \log n\right)$. We compute $\mathcal{A}(\mathcal{R})$, using the randomized incremental algorithm in [25], and return a point $\tilde{q} \in \mathbb{R}^{2}$ of maximum depth in $\mathcal{A}(\mathcal{R})$.

This completes the description of the algorithm.

Correctness We claim that $\omega(\tilde{q}, \mathcal{K}) \geq\left(1-\frac{1}{2} \delta\right) \omega(\mathcal{K})$. Indeed, let $q^{*} \in \mathbb{R}^{2}$ be, as above, a point of maximum depth in $\mathcal{A}(\mathcal{K})$. We apply (3), use the fact that $\omega\left(q^{*}, \mathcal{K}\right) \geq$ $\rho$, and consider two cases. If $\omega(\tilde{q}, \mathcal{K}) \geq \rho$ then 


$$
\begin{aligned}
\omega(\tilde{q}, \mathcal{K}) & \geq \frac{\omega(\tilde{q}, \mathcal{R})}{1+\frac{1}{4} \delta} \geq \frac{\omega\left(q^{*}, \mathcal{R}\right)}{1+\frac{1}{4} \delta} \geq \frac{1-\frac{1}{4} \delta}{1+\frac{1}{4} \delta} \omega\left(q^{*}, \mathcal{K}\right) \\
& \geq\left(1-\frac{1}{2} \delta\right) \omega\left(q^{*}, \mathcal{K}\right)=\left(1-\frac{1}{2} \delta\right) \omega(\mathcal{K}) .
\end{aligned}
$$

On the other hand, if $\omega(\tilde{q}, \mathcal{K})<\rho$ then

$$
\begin{aligned}
\omega(\tilde{q}, \mathcal{K}) & \geq \omega(\tilde{q}, \mathcal{R})-\frac{\delta}{4} \rho \geq \omega\left(q^{*}, \mathcal{R}\right)-\frac{\delta}{4} \rho \\
& \geq\left(1-\frac{\delta}{4}\right) \omega\left(q^{*}, \mathcal{K}\right)-\frac{\delta}{4} \rho \\
& \geq\left(1-\frac{\delta}{4}\right) \omega\left(q^{*}, \mathcal{K}\right)-\frac{\delta}{4} \omega\left(q^{*}, \mathcal{K}\right) \\
& =\left(1-\frac{1}{2} \delta\right) \omega(\mathcal{K}) .
\end{aligned}
$$

Hence in both cases the claim holds. As argued earlier, this implies the desired property

$$
\Phi(\tilde{q}, \mathcal{E}) \geq(1-\delta) \Phi(\mathcal{E})
$$

Running time We now analyze the expected running time of the algorithm. We first note that we do not have to compute the set $\mathcal{K}$ explicitly to obtain a random sample of $\mathcal{K}$. Indeed, a random racetrack can be chosen by first randomly choosing a segment $e_{i} \in \mathcal{E}$, and then by choosing (independently) a random racetrack of $\mathcal{K}_{i}$. Hence, each sample $\mathcal{R}_{i}$ can be constructed in $O\left(v_{i}\right)$ time, and the final sample $\mathcal{R}$ in $O(v)$ time.

To analyze the expected time taken by the $i$ th step of the first phase, we bound the expected number of vertices in $\mathcal{A}\left(\mathcal{R}_{i}\right)$.

Lemma 13 The expected number of vertices in the arrangement $\mathcal{A}\left(\mathcal{R}_{i}\right)$ is $O\left(2^{i} \log ^{3} n\right)$.

Proof By Lemma 12 if we perform the $i$ th step of the first phase then $\omega(\mathcal{K}) \leq \rho_{i-1}=$ $2 \rho_{i}$. Therefore, using (3) we have

$$
\omega\left(\mathcal{R}_{i}\right) \leq \omega(\mathcal{K})+2 \varepsilon \rho_{i} \leq 2 \rho_{i}+2 \varepsilon \rho_{i}<3 \rho_{i}
$$

Therefore, $\Delta\left(\mathcal{R}_{i}\right)=\omega\left(\mathcal{R}_{i}\right)\left|\mathcal{R}_{i}\right| \leq 3 \rho_{i} v_{i}=O(\log n)$. The elements in $\mathcal{R}_{i}$ are chosen from $\mathcal{K}$ using the 2 -stage random sampling mechanism described above, which we can rearrange so that we first choose a random sample $\mathcal{E}_{i}$ of segments, and then, with this choice fixed, we choose the random expansion distances. This allows us to view $\mathcal{R}_{i}$ as a set of racetracks over a fixed set $\mathcal{E}_{i}$ of segments, each of which is the Minkowski sum of a segment of $\mathcal{E}_{i}$ with a disk of a random radius, where the radii are drawn uniformly at random and independently from the set $\tilde{\varphi}$. There is a minor technical issue: we might choose in $\mathcal{E}_{i}$ the same segment $e \in \mathcal{E}$ several times, and these copies of $e$ are not pairwise-disjoint. To address this issue, we slightly shift these multiple copies of $e$ so as to make them pairwise-disjoint. Assuming that $\mathcal{E}$ is in general position and that the cdf defining $\varphi$ is in "general position" with respect to the locations of the segments of $\mathcal{E}$, as defined in Sect. 3, this will not affect the asymptotic maximum depth in the arrangement of the sample. 
By Corollary 1, applied under the density model and conditioned on a fixed choice of $\mathcal{E}_{i}$, the expected value of $\left|\mathcal{A}\left(\mathcal{R}_{i}\right)\right|$ is

$$
\mathrm{E}\left[\left|\mathcal{A}\left(\mathcal{R}_{i}\right)\right|\right]=O\left(\Delta\left(\mathcal{R}_{i}\right) v_{i} \log n\right)=O\left(2^{i} \log ^{3} n\right),
$$

implying the same bound for the unconditional expectation too.

The expected time spent in constructing $\mathcal{A}\left(\mathcal{R}_{i}\right)$ by the randomized incremental algorithm in [25] is $O\left(v_{i} \log v_{i}+\left|\mathcal{A}\left(\mathcal{R}_{i}\right)\right|\right)=O\left(2^{i} \log ^{3} n\right)$. Hence, the $i$ th step of the first phase takes $O\left(2^{i} \log ^{3} n\right)$ expected time. Summing this bound over the steps of the first phase, we conclude that the expected time spent in the first phase is $O\left(n \log ^{3} n\right)$.

In the second phase, $|\mathcal{R}|=O\left(\frac{1}{\delta^{2} \rho} \log n\right)=O\left(\frac{n}{\delta^{2}} \log n\right)$, and the same argument as above, using (3), implies that

$$
\omega(\mathcal{R}) \leq \max \left\{\left(1+\frac{\delta}{4}\right) \omega(\mathcal{K}), \omega(\mathcal{K})+\frac{\delta}{4} \rho\right\}=O(\rho) ;
$$

where the latter bound follows as $\omega(\mathcal{K}) \in[\rho, 2 \rho]$. Hence, $\Delta(\mathcal{R})=\omega(\mathcal{R}) \cdot|\mathcal{R}|=$ $O\left(\frac{1}{\delta^{2}} \log n\right)$, and the expected size of $\mathcal{A}(\mathcal{R})$ is thus $O(\Delta(\mathcal{R}) \cdot|\mathcal{R}| \log n)=O\left(\frac{n}{\delta^{4}} \log ^{3} n\right)$. Since this dominates the cost of the other steps in this phase, the second phase takes $O\left(\frac{n}{\delta^{4}} \log ^{3} n\right)$ expected time.

Putting everything together, we obtain that the expected running time of the procedure is $O\left(\frac{n}{\delta^{4}} \log ^{3} n\right)$, and it computes, with high probability, a point $\tilde{q}$ such that $\Phi(\tilde{q}, \mathcal{E}) \geq(1-\delta) \Phi(\mathcal{E})$. This completes the proof of Theorem 3 .

\section{Discussion}

We have shown that if we take the Minkowski sums of the members of a family of pairwise-disjoint convex sets, each of constant description complexity, with disks whose radii are randomly chosen according to a suitable probabilistic model, then the expected complexity of the union of the Minkowski sums is near linear. This generalizes the result of Kedem et al. [18] and indicates that the complexity of the union of Minkowski sums is quadratic only if the expansion distances are chosen in an adversarial manner. Our model is related to the so-called realistic input models, proposed to obtain more refined bounds on the performance of a variety of geometric algorithms [11]. There are also some similarities between our model and the framework of smoothed analysis [26].

A natural collection of open problems is to tighten the bounds in our theorems or prove corresponding lower bounds. In particular, the following questions arise. (i) The $O\left(n^{1+\varepsilon}\right)$ bound of Theorem 1 is unlikely to be tight. Is it possible to prove an $O(n \log n)$ upper bound as we did for polygons in Theorem 2? (ii) Can the bound in Theorem 2 be improved from $O\left(s^{2} n \log n\right)$ to $O(s n \log n)$ ? (iii) Is the bound of Theorem 2 asymptotically tight, even for segments, or could one prove a tighter $o(n \log n)$ bound? maybe even linear?

It would be useful to prove, or disprove, that the density and permutation models are equivalent, in the sense that the value of $\psi(\mathcal{C})$ is asymptotically the same under 
both models for any family $\mathcal{C}$ of pairwise-disjoint convex sets. Nevertheless, it is conceivable that there is a large class of density functions for which the density model yields a better upper bound.

Another interesting direction for future research is to explore other problems that can benefit from our model. For example, following the work in this paper, Har-Peled and Raichel [15] showed that the complexity of the multiplicatively weighted Voronoi diagram of $n$ points in the plane is $O(n$ polylog $n)$, if the weights are chosen randomly, in either of the two models assumed in this paper. Recall that if the weights are chosen by an adversary, then the complexity of the diagram can be quadratic in the worst case [7]. More recently, Chang et al. [9] showed a similar bound for more generalized Voronoi diagrams, where the distance to a site might depend on several parameters that are chosen randomly. We believe that the expected complexity of the multiplicativelyweighted Voronoi diagram of an arbitrary set of points in $\mathbb{R}^{3}$ is subquadratic if their weights are chosen using one of our models and the spread of the weights is at least a constant. Note that, in contrast, the standard Voronoi diagram can have quadratic complexity in the worst case.

Acknowledgments The authors thank Emo Welzl for useful discussions concerning the two probabilistic models used in the paper, and the anonymous referees for their valuable comments. A preliminary version of this paper appeared in Proc. 29th Annual Symposium of Computational Geometry, 2013, pp. 177-186. Work by Pankaj Agarwal and Micha Sharir has been supported by Grant 2012/229 from the U.S.-Israel Binational Science Foundation. Work by Pankaj Agarwal has also been supported by NSF under Grants CCF-09-40671, CCF-10-12254, and CCF-11-61359, by ARO Grant W911NF-08-1-0452, and by an ERDC contract W9132V-11-C-0003. Work by Sariel Har-Peled has been supported by NSF under Grants CCF0915984 and CCF-1217462. Work by Haim Kaplan has been supported by Grant 822/10 from the Israel Science Foundation, Grant 1161/2011 from the German-Israeli Science Foundation, and by the Israeli Centers for Research Excellence (I-CORE) program (Center No. 4/11). Work by Micha Sharir has also been supported by NSF Grant CCF-08-30272, by Grants 338/09 and 892/13 from the Israel Science Foundation, by the Israeli Centers for Research Excellence (I-CORE) program (Center No. 4/11), and by the Hermann Minkowski-MINERVA Center for Geometry at Tel Aviv University.

\section{References}

1. Agarwal, P.K., Efrat, A., Ganjugunte, S.K., Hay, D., Sankararaman, S., Zussman, G.: Network vulnerability to single, multiple, and probabilistic physical attacks. In: Proceedings of Military Communication Conference 2010, pp. 1824-1829 (2010)

2. Agarwal, P.K., Efrat, A., Ganjugunte, S. K., Hay, D., Sankararaman, S., Zussman, G.: The resilience of WDM networks to probabilistic geographical failures. In: IEEE/ACM Transactions of Network 21 pp. 1525-1538 (2013)

3. Agarwal, P.K., Ezra, E., Sharir, M.: Near-linear approximation algorithms for geometric hitting sets. Algorithmica 63, 1-25 (2012)

4. Agarwal, P. K., Hagerup, T., Ray, R., Sharir, M., Smid, M., Welzl, E.: Translating a planar object to maximize point containment. In: Proceedings of 10th Annual European Symposium on Algorithms, pp. 42-53 (2002)

5. Agarwal, P.K., Pach, J., Sharir, M.: State of the union (of geometric objects). In: Goodman, J., Pach, J., Pollack, R. (eds.) Surveys on Discrete and Computational Geometry, pp. 9-48. American Mathematical Society, Providence, RI (2008)

6. Aronov, B., Har-Peled, S.: On approximating the depth and related problems. SIAM J. Comput. 38, 899-921 (2008)

7. Aurenhammer, F., Klein, R.: Voronoi diagrams. In: Sack, J.-R., Urrutia, J. (eds.) Handbook of Computational Geometry, pp. 201-290. Elsevier, Amsterdam (1999)

8. Bhandari, R.: Survivable Networks: Algorithms for Diverse Routing. Kluwer, Norwell, MA (1998) 
9. Chang, H.-C., Har-Peled, S., Raichel, B.: From proximity to utility: a Voronoi partition of Pareto optima, CoRR. http://arxiv.org/abs/1404.3403 (2014)

10. Clarkson, K.L., Shor, P.W.: Applications of random sampling in computational geometry II. Discrete Comput. Geom. 4, 387-421 (1989)

11. de Berg, M., Katz, M.J., van der Stappen, A.F., Vleugels, J.: Realistic input models for geometric algorithms. Algorithmica 34, 81-97 (2002)

12. Edelsbrunner, H., Fasy, B.T., Rote, G.: Add isotropic Gaussian kernels at own risk: more and more resilient modes in higher dimensions. In: Proceedings of 28th Annual Symposium Computational Geometry, pp. 91-100 (2012)

13. Foster, J.S., Gjelde, E., Graham, W.R., Hermann, R.J., Kluepfel, H.M., Lawson, R.L., Soper, G.K., Wood, L.L., Woodard, J.B.: Report of the Commission to Assess the Threat to the United States from Electromagnetic Pulse (EMP) Attack, Critical National Infrastructures (2008)

14. Graham, R.L., Knuth, D.E., Patashnik, O.: Concrete Mathematics: A Foundation for Computer Science, 2nd edn. Addison Wesley, Boston (1994)

15. Har-Peled, S., Raichel, B.: On the complexity of randomly weighted Voronoi diagrams. In: Proceedings of 30th Annual Symposium Computational Geometry, pp. 232-241 (2014)

16. Har-Peled, S., Sharir, M.: Relative $(p, \varepsilon)$-approximations in geometry. Discrete Comput. Geom. 45, 462-496 (2011)

17. Johnson, N.L., Kemp, A.W., Kotz, S.: Univariate Discrete Distributions, 3rd edn. Wiley, New York (2005)

18. Kedem, K., Livne, R., Pach, J., Sharir, M.: On the union of Jordan regions and collision-free translational motion amidst polygonal obstacles. Discrete Comput. Geom. 1, 59-71 (1986)

19. Lifshitz, L.M., Pizer, S.M.: A multiresolution hierarchical approach to image segmentation based on intensity extrema. IEEE Trans. Pattern Anal. Mach. Intell. 12, 529-540 (1990)

20. Matoušek, J.: Lectures on Discrete Geometry. Springer, Heidelberg (2002)

21. Neumayer, S., Modiano, E.: Network reliability with geographically correlated failures. In: Proceedings 29th IEEE International Conference on Computer Communications, pp. 1658-1666 (2010)

22. Neumayer, S., Zussman, G., Cohen, R., Modiano, E.: Assessing the vulnerability of the fiber infrastructure to disasters. IEEE/ACM Trans. Netw. 19, 1610-1623 (2011)

23. Ou, C., Mukherjee, B.: Survivable Optical WDM Networks. Springer, Heidelberg (2005)

24. Pach, J., Sharir, M.: On the boundary of the union of planar convex sets. Discrete Comput. Geom. 21, 321-328 (1999)

25. Sharir, M., Agarwal, P.K.: Davenport-Schinzel Sequences and Their Geometric Applications. Cambridge University Press, Cambridge (1995)

26. Spielman, D.A., Teng, S.H.: Smoothed analysis: an attempt to explain the behavior of algorithms in practise. Commun. ACM 52, 76-84 (2009)

27. Wu, W., Moran, B., Manton, J., Zukerman, M.: Topology design of undersea cables considering survivability under major disasters. In: Proceedings of International Conference on Advanced Information Networking and Applications Workshops, pp. 1154-1159 (2009) 